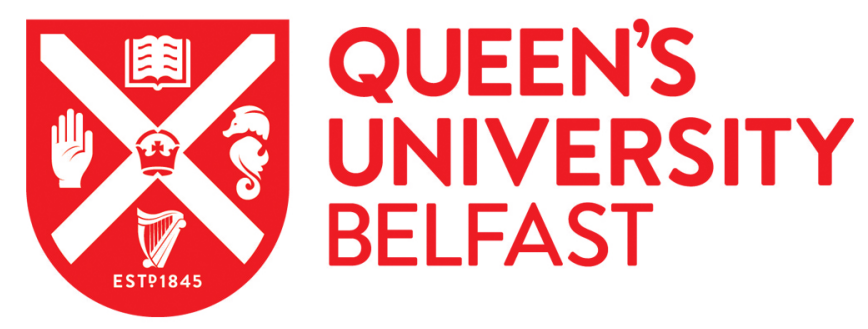

\title{
Robust control for vibration control systems with dead-zone band and time delay under severe disturbance using adaptive fuzzy neural network
}

Do, X. P., \& Van, M. (2020). Robust control for vibration control systems with dead-zone band and time delay under severe disturbance using adaptive fuzzy neural network. Journal of the Franklin Institute, 357(17), 1228112307. https://doi.org/10.1016/j.jfranklin.2020.09.011

Published in:

Journal of the Franklin Institute

Document Version:

Peer reviewed version

Queen's University Belfast - Research Portal:

Link to publication record in Queen's University Belfast Research Portal

\begin{abstract}
Publisher rights
(C) 2020 The Franklin Institute.

This manuscript is distributed under a Creative Commons Attribution-NonCommercial-NoDerivs License

(https://creativecommons.org/licenses/by-nc-nd/4.0/), which permits distribution and reproduction for non-commercial purposes, provided the author and source are cited.

\section{General rights}

Copyright for the publications made accessible via the Queen's University Belfast Research Portal is retained by the author(s) and / or other copyright owners and it is a condition of accessing these publications that users recognise and abide by the legal requirements associated with these rights.
\end{abstract}

\section{Take down policy}

The Research Portal is Queen's institutional repository that provides access to Queen's research output. Every effort has been made to ensure that content in the Research Portal does not infringe any person's rights, or applicable UK laws. If you discover content in the

Research Portal that you believe breaches copyright or violates any law, please contact openaccess@qub.ac.uk. 
FI-D-20-00299.R1

\title{
Robust control for vibration control systems with dead-zone band and time delay under severe disturbance using adaptive fuzzy neural network
}

\begin{abstract}
Do Xuan Phu' ${ }^{*}$, Van Mien ${ }^{2 *}$
${ }^{1}$ MediRobotics Laboratory, Department of Mechatronics and Sensor Systems Technology, Vietnamese-German University, Binh Duong, Vietnam. Email: phu.dx@vgu.edu.vn ${ }^{2}$ School of Electronics, Electrical Engineering and Computer Science, Queen's University Belfast, BT71NN, UK. Email: m.van@qub.ac.uk
\end{abstract}

"Corresponding authors: Dr. Do Xuan Phu (Email: phu.dx@vgu.edu.vn) and Dr. Van Mien (Email:m.van@qub.ac.uk) 


\begin{abstract}
This study proposes a novel adaptive control method to deal with the dead-zone and time delay issues in actuators of vibration control systems. The controller is formulated based on a type-2 fuzzy neural network integrating with a new modification of Riccati-like equation. The developed new type Riccati-like equation is significant as it reduces energy consumption of control inputs to minimum. Two approaches are suggested to improve performance of the system using the basic elements of Riccati equation. In addition, a fuzzy neural network is applied to approximate the unmodeled dynamics and a sliding mode controller is developed to enhance the robustness of the system against uncertainties and disturbances. After proving the stability of the proposed controller via Lyapunov criterion, the effectiveness of the proposed approach is validated based on computer simulation for vibration control of a vehicle seat suspension. It is demonstrated that the unwanted vibrations due to external excitations are well controlled despite of the presence of dead-zone and time delay in actuators. Furthermore, when comparing with other two state-of-theart robust controllers $[23,36]$, the proposed controller provides better vibration suppression capacity and requires less energy consumption.
\end{abstract}

Keywords: Type 2-fuzzy neural network, adaptive control, neural network, dead-zone, time delay, MR damper, vibration control 


\section{Introduction}

Developing new control methodologies for modern devices attracts a lot of attentions from both academia and industry because it is important but yetyet challenging. In general, the control approaches for modern devices can be classified inte:into classical control and modern control. The goal of development of new control mechanism is to satisfy multiple requirements of consumer such as comfortable, human ergonomics, vibration and disturbance suppression, high precision, and so forth. Many great efforts have been spent on designing new control schemes to achieve that goals. For instance, an adaptive control using a super-twisting integrated with adecoupled-terminal sliding mode control was presented in [1], where the sliding surface was designed based on a terminal sliding surface and a super-twisting controller was employed to eliminate chattering. An integral sliding mode control with prescribed performance was developed in [2]. In this approach, a tanh function was used to obtain smooth control efforts and a prescribed function was utilized to ensure the tracking errors are limited within the desirable boundaries. In the design of control system, besides considering disturbances, the dead-zone in actuators and unmodeled dynamics are also significant that need to be taken inte accountconsidered. A methodology to deal with the dead-zone input and unmodeled dynamics was recently studied in [3], where the dead-zone was simply defined as hysteretic properties of the system and the unmodeled dynamics was assumed to be bounded by some constants. Due to its approximation capability, fuzzy logic system (FLS) has been frequently utilized in many practical systems subjected to unmodeled dynamics, parameter variations and non-predictable nonlinear parameters-, i.e.g., actuators and sensor faults. For example, a back-stepping designbased fuzzy approximation has been developed to deal with actuator failures and unknown system dynamics [4]. Moreover, in order to enhance system robustness against external disturbances and input constraints, an adaptive controller was integrated with an $H$-infinity technique [5], in which the constraint inputs are encoded into $H$-infinity performance. Recently, various types of robust controllers have been proposed by combining two or more different controllers to increase the system robustness against the diverse uncertainties and unmodeled dynamics. In general, the stability of these composite or hybrid controllers can be proven based on Lyapunov criterion [6]. A hybrid controller based on PI control, sliding mode control and radial basic function neural 
network was proposed in [7]. A problem associated with gain adaptation of conventional sliding mode control was described in [8]. An integration between nonsingular fast terminal sliding model controller and PID controller was also applied to design adaptive controller in [9]. A new two-step distributed adaptive leader-following consensus control of connected Lagrangian systems was described in [10]. A continuous sliding mode control law coupled with an adaptive sliding mode observer was presented in [11]. The nonsingular terminal sliding surface used in [11] was similar to that of the one used in [9]. A study of disturbance rejection based on sliding mode control with prescribed performance for robot manipulators was carried out in [12]. In [13], a PID controller was combined with an $H$-infinity controller based on Riccati-like equation. It is notice that the Riccati-like equation plays an important role in connecting a controller with other controllers without loss of the stability. Therefore, it is often employed for deriving hybrid controllers, which comprise more than two different controllers. Recently, the $\underline{\text { a }}$ static output feedback control for compensating faults of switched system was presented in [14].

In this model [14], the fuzzy control is applied for finding properties of the switched system, and conditions for faults were designed based on the switched nonlinear systems is converted into a switched fuzzy system, and the stability of the system is solved using a linear matrix inequality method._A similar method [14] was also studied in [15] taking the, but for missing measurements problem of the system states of the system into consideration. Fault tolerant control under state/noise estimation was studied in [16] using the. The linear matrix inequality approachwas also used for setup conditions [16]. In [17], the-a fault tolerant control method [17] was proposed by an adaptation law with matrices relating to ed-Hurwitz definition.

Most of actuators in practical applications possess a dead-zone band. Violations of the control inputs with the dead-zone will degrade the system performance significantly. Moreover, time delay of actuators provides adverse effects on system performance. These effects become even much worst for complex or composite control systems, which integrate different controller types and feedback loops. In order to reduce the adverse effects due to the dead-zone band, many approaches have been developed for different types of applications [18-23]. For example, an adaptive fuzzy controller integrated with an $H$-infinity technique was utilized to solve the unknown dead-zone output in [18]. Neural networks approach for the dead-zone issue was studied 
in [19]. The dead-zone model was considered as an additional disturbance component and an adaptive robust control was designed to eliminate the effects of dead-zone in [20, 21]. In [22], a modification of backstepping control technique with prescribed performance was proposed to deal with the dead-zone input for air-breathing hypersonic vehicles. An adaptive fuzzy control combined with a modification of Riccati-like equation was studied in [23]. However, this approach have has not considered the dead-zone effects in the design.

From the above literature survey, the fuzzy logic system is usually selected as an effective tool for controlling the system subjected to several uncertainties including unmodeled dynamics and parameter variations. This choice is reasonable because the fuzzy logic system can act like a filter in the design of robust control mechanism. Besides the classical fuzzy model, namely type 1 model, the type 2 model has also been employed in the design of control systems [24, 25, 27-30]. Type-2 fuzzy sets and systems generalize standard Type-1 fuzzy sets and systems so that more uncertainty can be handled. Because large data is used to establish the fuzzy rules, clustering method is normally used as the first step to cluster dataset. The FLS and neural network have their own advantages and disadvantages in terms of nonlinear function approximation, so combining FLS and neural network will likely bring more merits while reducing their disadvantages for uncertain nonlinear systems.

Motivated by the aforementioned discussions, in this work, a type 2 fuzzy model associated with a neural network is adopted for modeling system dynamics. The modeled dynamics is then integrated with an adaptive sliding mode controller to improve the system robustness against the system uncertainties and disturbances, which includes both dead-zone input and time delay of the actuators. In summary, the major contributions of this paper can be highlighted as follows:

(1) A novel adaptive law for dealing with the dead-zone and delayed timetime delay phenomenon is proposed.

(2) A new modification of Riccati-like equation for both dead-zone phenomenon and time delay of actuators is suggested.

(3) The main parameter, which decide how to save related saving energy consumption of the control system, is pointed out in the adaptation laws. 
(4) Compared with other state-of-the-art methods [23, 36], the proposed approach provides superior performance.

\section{Design of Adaptive Fuzzy Control with Actuator Dead-zone and Time Delay}

\subsection{Interval Type 2 Fuzzy Model}

In this study, the interval type 2 fuzzy neural networks (IT2FNN) model [24,25,27-30] is employed. Before applying, the clustering progress [26] is carried out to decrease the volume of data, and then the rule base of IT2FNN is defined. The jth If-Then rule can be expressed as follows:

$R_{f}{ }^{j}:$ If $h_{1}$ is $\boldsymbol{H}_{f 1}^{j}$ and $\ldots$ and $h_{n}$ is $\boldsymbol{H}_{f n}^{j}$ Then $g$ is $a_{0}^{j}+\sum_{i=1}^{n} a_{i}^{j} h_{i}$

where, $\boldsymbol{H}_{f i}^{j}(i=1, \ldots, n ; j=1, \ldots, m)$ are fuzzy sets, $m$ is the number of rules, and $a_{i}^{j}$ are interval sets.

The defuzzified output is determined by

$g_{f}=\frac{g_{l}+g_{r}}{2}=\frac{\mathbf{\Xi}_{l}^{T} \boldsymbol{\Phi}_{l}^{f}+\mathbf{\Xi}_{r}^{T} \boldsymbol{\Phi}_{r}^{f}}{2}$

In equation (2), $\mathbf{\Xi}_{l}^{T}=\left[w_{1}^{l} w_{2}^{l} w_{3}^{l} \ldots w_{n}^{l}\right]$ and $\boldsymbol{\Xi}_{r}^{T}=\left[\begin{array}{lll}w_{1}^{r} & w_{2}^{r} & w_{3}^{r} \ldots w_{n}^{r}\end{array}\right]$ are the weighting vectors, which symbolize the relation between the rule layer and type-reduction. The weights firing strength vectors are given by

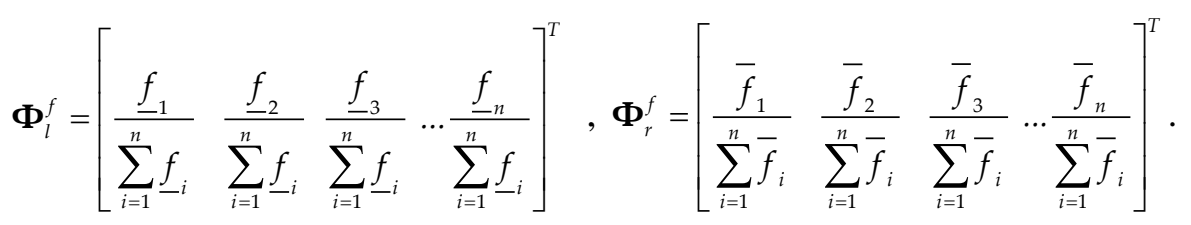

\subsection{Adaptive Fuzzy Control with Actuator Dead-zone and Time Delay}

In order to formulate a new composite controller, a single-input single-output (SISO) nonlinear system governed by the following equation is considered:

$\dot{\mathbf{x}}=\mathbf{f}(\mathbf{x})+\mathbf{g}(\mathbf{x}) \phi_{u}(t)+\Lambda^{T} \mathbf{x}_{\tau}+\mathbf{d}(\mathbf{t})$

where, $\mathbf{f}(\mathbf{x}) \in R^{n}$ and $\mathbf{g}(\mathbf{x}) \in R^{n}$ are two unknown non-linear function vectors, $\phi_{u}(t) \in R^{1}$ is control function, $\mathbf{d}(t) \in R^{n} \quad$ is an external disturbance vector, 
$\mathbf{x}=\left[x_{1}, x_{2}, \ldots, x_{n}\right]^{T}=\left[x_{1}, \dot{x}_{1}, \ldots, x_{1}^{(n-1)}\right]^{T} \in R^{n} \quad$ is the state vector of the system, $\mathbf{x}_{\tau}=\left[\begin{array}{llll}x_{1}^{\tau}\left(t-\tau_{1}\right) & x_{2}^{\tau}\left(t-\tau_{2}\right) & \ldots & x_{n}^{\tau}\left(t-\tau_{n}\right)\end{array}\right]^{T} \quad$ is the state with time delay, $\Lambda=\left(\begin{array}{ccc}\Lambda_{1}(t) & & 0 \\ & \ddots & \\ 0 & & \Lambda_{n}(t)\end{array}\right), \Lambda_{m}(t)=\left[\begin{array}{ll}z_{m}(t) & 0\end{array}\right]^{T}$ is the nonlinear function, which relates timevarying of the system with an assumed boundary $\left|z_{m}(t)\right| \leq \delta z_{m}$, where $\delta z_{m}$ is an unknown positive constant.

An equivalent form of the system (3) can be expressed as follows:

$\dot{\mathbf{x}}=\mathbf{A} \mathbf{x}+\mathbf{B}\left[\mathbf{f}(\mathbf{x})+\mathbf{g}(\mathbf{x}) \phi_{u}(t)+\mathbf{\Lambda}^{T} \mathbf{x}_{\tau}+\mathbf{d}(t)\right]$

where, $\mathbf{A}=\left[\begin{array}{cccc}0 & 1 & \cdots & 0 \\ 0 & 0 & \cdots & 0 \\ \vdots & \vdots & \cdots & \vdots \\ 0 & 0 & \cdots & 1 \\ 0 & 0 & \cdots & 0\end{array}\right], \mathbf{B}=\left[\begin{array}{c}0 \\ 0 \\ \vdots \\ 1\end{array}\right]$.

The control function $\phi_{u}(t)$ in (4) can be designed as follows:

$\phi_{u}(t)=\beta u+\varphi(u)+\delta(\dot{u})$

where, $\beta$ is the parameter related to the slope of the dead-zone performance, as shown in Figure $1, u$ is the control input, and the functions $\varphi(u)$ and $\delta(\dot{u})$ are defined as follows [19]:

$$
\begin{aligned}
& \varphi(u)=\left\{\begin{array}{l}
-\beta \theta^{+} \quad \text { for } u \geq \theta^{+} \\
-\beta u \text { for } \theta^{-} \leq u \leq \theta^{+} \\
-\beta \theta^{-} \quad \text { for } u \leq \theta^{-}
\end{array}\right. \\
& \delta(\dot{u})=\beta|\dot{u}| \operatorname{sign}(\dot{u})
\end{aligned}
$$

where, $\beta$ is a chosen constant gain. The values of Eqs. (6) and (7) are assumed to be bounded by $|\varphi(u)| \leq \Delta \varphi$ and $|\delta(\dot{u})| \leq \Delta \delta$, respectively. The tracking reference function is determined as: $\dot{\mathbf{x}}_{d}=\mathbf{A} \mathbf{x}_{d}+\mathbf{B} \ddot{\mathbf{x}}_{d}$

where, $\mathbf{x}_{d}=\left[x_{d 1}, x_{d 2}, \ldots, x_{d n}\right]^{T}=\left[x_{d 1}, \dot{x}_{d 1}, \ldots, x_{d 1}^{(n-1)}\right]^{T} \in R^{n}$. The error dynamic equation is defined as $\mathbf{e}=\mathbf{x}-\mathbf{x}_{d}$. The derivative of this error can be calculated as:

$$
\dot{\mathbf{e}}=\dot{\mathbf{x}}-\dot{\mathbf{x}}_{d}=\mathbf{A x}+\mathbf{B}\left[\mathbf{f}(\mathbf{x})+\mathbf{g}(\mathbf{x}) \phi_{u}(t)+\mathbf{\Lambda}^{T} \mathbf{x}_{\tau}+\mathbf{d}(t)\right]-\left(\mathbf{A} \mathbf{x}_{d}+\mathbf{B} \ddot{\mathbf{x}}_{d}\right)
$$


$\Leftrightarrow \dot{\mathbf{e}}=\mathbf{A}\left(\mathbf{x}-\mathbf{x}_{d}\right)+\mathbf{B}\left[\mathbf{f}(\mathbf{x})+\mathbf{g}(\mathbf{x}) \phi_{u}(t)+\mathbf{\Lambda}^{T} \mathbf{x}_{\tau}+\mathbf{d}(t)-\ddot{\mathbf{x}}_{d}\right]$

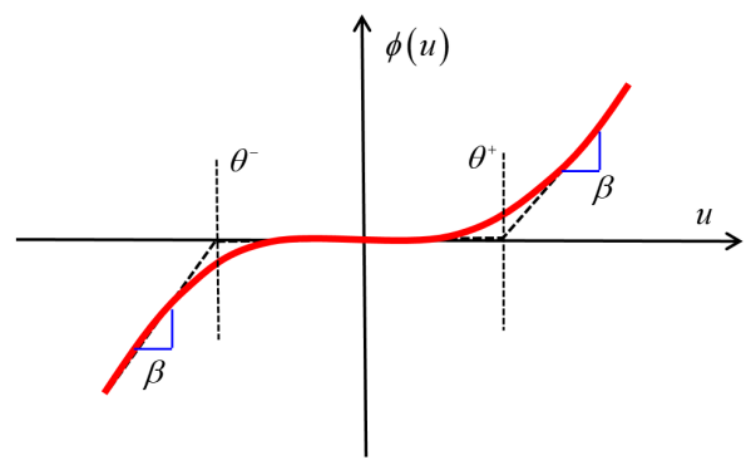

Figure 1. Nonlinear dead-zone band model.

From Eq. (9), a new feedback gain matrix $\mathbf{K}=\operatorname{diag}\left\{\begin{array}{lll}\mathbf{K}_{1} & \cdots & \mathbf{K}_{n}\end{array}\right\}$ with $\mathbf{K}_{i}=\left[\begin{array}{ll}K_{i 1} & K_{i 2}\end{array}\right]^{T}$ is a chosen vector such that all roots of the characteristic polynomial of $\mathbf{A}_{i}=\mathbf{A}-\mathbf{B K}_{i}^{T}, i=1, \ldots, n$ are in the open left-half plane. From this definition, a new matrix $\mathbf{A}_{\Delta}=\operatorname{diag}\left\{\begin{array}{lll}\mathbf{A}_{1} & \cdots & \mathbf{A}_{n}\end{array}\right\}$ is declared. Eq. (9) is then rewritten as follows:

$\dot{\mathbf{e}}=\mathbf{A e}-\mathbf{B K} \mathbf{K}^{T} \mathbf{e}+\mathbf{B}\left[\mathbf{K}^{T} \mathbf{e}+\mathbf{f}(\mathbf{x})+\mathbf{g}(\mathbf{x})[\beta u+\varphi(u)+\delta(\dot{u})]+\mathbf{\Lambda}^{T} \mathbf{x}_{\tau}+\mathbf{d}(t)-\ddot{\mathbf{x}}_{d}\right]$

$\Leftrightarrow \dot{\mathbf{e}}=\mathbf{A}_{\Delta} \mathbf{e}+\mathbf{B}\left[\mathbf{K}^{T} \mathbf{e}+\mathbf{f}(\mathbf{x})+\mathbf{g}(\mathbf{x})[\beta u+\varphi(u)+\delta(\dot{u})]+\mathbf{\Lambda}^{T} \mathbf{x}_{\tau}+\mathbf{d}(t)-\ddot{\mathbf{x}}_{d}\right]$

Using the result of fuzzy neural networks model (2), the nonlinear functions $\mathbf{f}(\mathbf{x})$ and $\mathbf{g}(\mathbf{x})$ can be expressed as:

$\hat{\mathbf{f}}(\mathbf{x})=\boldsymbol{\Xi}_{f}(x) \boldsymbol{\Phi}_{f}, \hat{\mathbf{g}}(\mathbf{x})=\boldsymbol{\Xi}_{g}(x) \boldsymbol{\Phi}_{g}$

When the fuzzy neural networks model is used, the minimum approximation errors of $\mathbf{f}(\mathbf{x})$ and $\mathbf{g}(\mathbf{x})$ are defined as follows:

$\Delta \mathbf{f}(\mathbf{x})=\mathbf{f}(\mathbf{x})-\hat{\mathbf{f}}\left(\mathbf{x} \mid \Phi_{f}^{*}\right), \Delta \mathbf{g}(\mathbf{x})=\mathbf{g}(\mathbf{x})-\hat{\mathbf{g}}\left(\mathbf{x} \mid \Phi_{g}^{*}\right)$

where, $\Delta \mathbf{f}(\mathbf{x})$ and $\Delta \mathbf{g}(\mathbf{x})$ are the minimum approximation errors. These errors will be compensated by using adaptation laws. The vectors $\boldsymbol{\Phi}_{f}^{*}$ and $\boldsymbol{\Phi}_{g}^{*}$ are defined as follows:

$\boldsymbol{\Phi}_{f}^{*}=\underset{\boldsymbol{\Phi}_{f} \in \Omega_{f}}{\arg \min }\left\{\sup _{\mathbf{x} \in \Lambda x}\left|\mathbf{f}(\mathbf{x})-\hat{\mathbf{f}}\left(\mathbf{x} \mid \boldsymbol{\Phi}_{f}\right)\right|\right\}, \boldsymbol{\Phi}_{g}^{*}=\underset{\boldsymbol{\Phi}_{g} \in \Omega_{g}}{\arg \min }\left\{\sup _{\mathbf{x} \in \Lambda x}\left|\mathbf{g}(\mathbf{x})-\hat{\mathbf{g}}\left(\mathbf{x} \mid \boldsymbol{\Phi}_{g}\right)\right|\right\}$

To support the next analysis steps, two new definitions are introduced as follows:

$\tilde{\boldsymbol{\Phi}}_{f}=\boldsymbol{\Phi}_{f}^{*}-\boldsymbol{\Phi}_{f}, \tilde{\boldsymbol{\Phi}}_{g}=\boldsymbol{\Phi}_{g}^{*}-\boldsymbol{\Phi}_{g}$

Using the results in Eqs. (11) and (14), Eq. (10) is rewritten as follows: 


$$
\begin{aligned}
& \dot{\mathbf{e}}=\mathbf{A}_{\Delta} \mathbf{e}+\mathbf{B}\left[\begin{array}{l}
\left.\mathbf{K}^{T} \mathbf{e}+\mathbf{f}(\mathbf{x})-\hat{\mathbf{f}}(\mathbf{x})+\hat{\mathbf{f}}(\mathbf{x})+(\mathbf{g}(\mathbf{x})-\hat{\mathbf{g}}(\mathbf{x})+\hat{\mathbf{g}}(\mathbf{x}))[\beta u+\varphi(u)+\delta(\dot{u})]+\right] \\
+\boldsymbol{\Lambda}^{T} \mathbf{x}_{\tau}+\mathbf{d}(t)-\ddot{\mathbf{x}}_{d}
\end{array}\right] \\
& \Leftrightarrow \dot{\mathbf{e}}=\mathbf{A}_{\Delta} \mathbf{e}+\mathbf{B}\left[\begin{array}{l}
\left.\mathbf{K}^{T} \mathbf{e}+\mathbf{\Xi}_{f}^{T}(x) \tilde{\mathbf{\Phi}}_{f}+\hat{\mathbf{f}}(\mathbf{x})+\left(\boldsymbol{\Xi}_{g}^{T}(x) \tilde{\mathbf{\Phi}}_{g}+\hat{\mathbf{g}}(\mathbf{x})\right)[\beta u+\varphi(u)+\delta(\dot{u})]+\right] \\
+\mathbf{\Lambda}^{T} \mathbf{x}_{\tau}+\mathbf{d}(t)-\ddot{\mathbf{x}}_{d}
\end{array}\right] \\
& \Leftrightarrow \dot{\mathbf{e}}=\mathbf{A}_{\Delta} \mathbf{e}+\mathbf{B}\left[\begin{array}{l}
\mathbf{K}^{T} \mathbf{e}+\mathbf{\Xi}_{f}^{T}(x) \tilde{\mathbf{\Phi}}_{f}+\hat{\mathbf{f}}(\mathbf{x})+\mathbf{\Xi}_{g}^{T}(x) \tilde{\mathbf{\Phi}}_{g}[\beta u+\varphi(u)+\delta(\dot{u})]+ \\
+\hat{\mathbf{g}}(\mathbf{x})[\beta u+\varphi(u)+\delta(\dot{u})]+\boldsymbol{\Lambda}^{T} \mathbf{x}_{\tau}+\mathbf{d}(t)-\ddot{\mathbf{x}}_{d}+\mathbf{D}
\end{array}\right]
\end{aligned}
$$

where, $\mathbf{D}=\boldsymbol{\Delta} \mathbf{f}+\Delta \mathbf{g}[\beta u+\varphi(u)+\delta(\dot{u})]$ is the minimum approximation error associated to deadzone phenomenon. The proposed controllers are now described as below.

\section{Proposed Control 1.}

In this proposed control 1, the state with time delay $\mathbf{x}_{\tau}=\left[\begin{array}{llll}x_{1}^{\tau}\left(t-\tau_{1}\right) & x_{2}^{\tau}\left(t-\tau_{2}\right) & \ldots & x_{n}^{\tau}\left(t-\tau_{n}\right)\end{array}\right]^{T}$ is neglected. The main control $u$ is determined as follows:

$$
u=u_{1}+u_{2}+u_{3}=\sum_{i=1}^{3} u_{i}
$$

where, $u_{1}$ is the equivalent control, $u_{2}$ is the first robustness control related to the Riccati-like equation, and $u_{3}$ is the second robustness control related to the dead-zone band and the minimum approximation error $\mathbf{D}$ of the system. These control inputs are designed as follows:

$$
\begin{aligned}
& u_{1}=-\frac{1}{\beta^{2} \hat{\mathbf{g}}(\mathbf{x})}\left(\hat{\mathbf{f}}(\mathbf{x})-\ddot{\mathbf{x}}_{d}+\mathbf{K}^{T} \mathbf{e}\right) \\
& u_{2}=-\frac{1}{\beta^{2} \hat{\mathbf{g}}(\mathbf{x})} \mathbf{R}^{-1} \mathbf{B}^{T} \mathbf{P e} \\
& u_{3}=-\frac{1}{\beta_{\text {min }}}(\Delta \varphi+\Delta \delta) \operatorname{sign}(\hat{\mathbf{g}}(\mathbf{x}))-\frac{1}{\beta^{2} \hat{\mathbf{g}}(\mathbf{x})} \mathbf{D}
\end{aligned}
$$

where, $\Delta \varphi$ is the upper bound value of $\|\varphi(u)\|, \Delta \delta$ is the upper bound value of $\|\delta(\dot{u})\|, \beta_{\text {min }}$ is the parameter related to the dead-zone model. The balance Riccati-like equation is given by

$$
\mathbf{P A}+\mathbf{A}^{T} \mathbf{P}+\mathbf{Q}+\mathbf{P B}\left(\frac{2}{\rho^{2}} \mathbf{I}-\beta_{\min } \mathbf{R}^{-1}\right) \mathbf{B}^{T} \mathbf{P}=0
$$

where, $0<\rho<1, \mathbf{I}$ is the identity matrix $(n \times n), \mathbf{Q}=\mathbf{Q}^{T}, \mathbf{R}$ denotes the robust $H$-infinity gain.

Theorem 1. The stability and robustness of the proposed controller for the dead-zone system are guaranteed if the control function $u$ is design as in (16) with its elements are implemented as in Eqs.(17), (18), (19), and the adaptation laws are given below. 


$$
\begin{aligned}
& \dot{\tilde{\mathbf{\Phi}}}_{f}^{T}=-\gamma_{f} \mathbf{\Xi}_{f}^{T} \mathbf{B}^{T} \mathbf{P e} \\
& \dot{\tilde{\mathbf{\Phi}}}_{g}^{T}=-\gamma_{g} \mathbf{\Xi}_{g}^{T} \mathbf{B}^{T} \mathbf{P e}(u+\Delta \varphi+\Delta \delta) \\
& \dot{\tilde{\mathbf{D}}}_{\beta}^{T}=-\frac{\gamma_{D}}{\beta} \mathbf{B}^{T} \mathbf{P e} \\
& \dot{\tilde{\beta}}_{\beta}=-\gamma_{\beta}\left[\mathbf{e}^{T} \mathbf{P B}\left(\hat{\mathbf{f}}(\mathbf{x})-\ddot{\mathbf{x}}_{d}+\mathbf{K}^{T} \mathbf{e}\right)\right]
\end{aligned}
$$

where, $\gamma_{f}, \gamma_{g}, \gamma_{D}, \gamma_{\beta}$ are chosen constants. It is noted that $\tilde{\beta}_{\beta}=\beta_{\beta}-\hat{\beta}_{\beta}, \beta_{\beta}=\frac{1}{\beta}, \hat{\beta}_{\beta}=\frac{1}{\beta^{2}}$, $\hat{\mathbf{D}}_{\beta}=\frac{1}{\beta} \mathbf{D}, \tilde{\mathbf{D}}_{\beta}=\mathbf{D}-\hat{\mathbf{D}}_{\beta} ; \gamma_{f}, \gamma_{g}, \gamma_{\beta}, \gamma_{D}$ are chosen constants

Proof: To prove the theorem 1, a Lyapunov function candidate is proposed as follows:

$$
L_{v}=\frac{1}{2 \beta} \mathbf{e}^{T} \mathbf{P e}+\frac{1}{2 \beta \gamma_{f}} \tilde{\boldsymbol{\Phi}}_{f}^{T} \tilde{\boldsymbol{\Phi}}_{f}+\frac{1}{2 \beta \gamma_{g}} \tilde{\boldsymbol{\Phi}}_{g}^{T} \tilde{\boldsymbol{\Phi}}_{g}+\frac{1}{2 \gamma_{\beta}} \tilde{\beta}_{\beta}^{2}+\frac{1}{2 \gamma_{D}} \tilde{\mathbf{D}}_{\beta}^{T} \tilde{\mathbf{D}}_{\beta}
$$

where $\tilde{\beta}_{\beta}=\beta_{\beta}-\hat{\beta}_{\beta}, \beta_{\beta}=\frac{1}{\beta}, \hat{\beta}_{\beta}=\frac{1}{\beta^{2}}, \hat{\mathbf{D}}_{\beta}=\frac{1}{\beta} \mathbf{D}, \tilde{\mathbf{D}}_{\beta}=\mathbf{D}-\hat{\mathbf{D}}_{\beta} ; \gamma_{f}, \gamma_{g}, \gamma_{\beta}, \gamma_{D}$ are chosen constants. The derivative of Eq. (26) is obtained as:

$$
\dot{L}_{v}=\frac{1}{2 \beta} \dot{\mathbf{e}}^{T} \mathbf{P e}+\frac{1}{2 \beta} \mathbf{e}^{T} \mathbf{P} \dot{\mathbf{e}}+\frac{1}{\beta \gamma_{f}} \dot{\tilde{\mathbf{\Phi}}}_{f}^{T} \tilde{\mathbf{\Phi}}_{f}+\frac{1}{\beta \gamma_{g}} \dot{\tilde{\mathbf{\Phi}}}_{g}^{T} \tilde{\mathbf{\Phi}}_{g}+\frac{1}{\gamma_{\beta}} \dot{\tilde{\beta}}_{\beta} \tilde{\beta}_{\beta}+\frac{1}{\gamma_{D}} \dot{\tilde{\mathbf{D}}}_{\beta}^{T} \tilde{\mathbf{D}}_{\beta}
$$

Using the results in Eq.(20), Eq.(27) is rewritten as follows:

$$
\begin{aligned}
\dot{L}_{v}= & \frac{1}{2 \beta} \mathbf{e}^{T}\left(\mathbf{A}^{T} \mathbf{P}+\mathbf{P A}\right) \mathbf{e}+\frac{1}{\beta} \mathbf{e}^{T} \mathbf{P B} \boldsymbol{\Xi}_{f}^{T} \tilde{\mathbf{\Phi}}_{f}+\frac{1}{\beta} \mathbf{e}^{T} \mathbf{P B K} \mathbf{K}^{T} \mathbf{e}+\frac{1}{\beta} \mathbf{e}^{T} \mathbf{P B} \mathbf{\Xi}_{g}^{T} \tilde{\mathbf{\Phi}}_{g}(\beta u+\Delta \varphi+\Delta \delta)+ \\
& +\frac{1}{\beta} \mathbf{e}^{T} \mathbf{P B} \hat{\mathbf{f}}(\mathbf{x})+\frac{1}{\beta} \mathbf{e}^{T} \mathbf{P} \mathbf{B} \hat{\mathbf{g}}(\mathbf{x})(\beta u+\Delta \varphi+\Delta \delta)+\frac{1}{\beta} \mathbf{e}^{T} \mathbf{P B d}+\frac{1}{\beta} \mathbf{e}^{T} \mathbf{P B D}-\frac{1}{\beta} \mathbf{e}^{T} \mathbf{P B} \ddot{\mathbf{x}}_{d}+ \\
& +\frac{1}{\beta \gamma_{f}} \dot{\tilde{\mathbf{\Phi}}}_{f}^{T} \tilde{\mathbf{\Phi}}_{f}+\frac{1}{\beta \gamma_{g}} \dot{\tilde{\mathbf{\Phi}}}_{g}^{T} \tilde{\mathbf{\Phi}}_{g}+\frac{1}{\gamma_{\beta}} \dot{\tilde{\boldsymbol{\beta}}}_{\beta} \tilde{\beta}_{\beta}+\frac{1}{\gamma_{D}} \dot{\tilde{\mathbf{D}}}_{\beta}^{T} \tilde{\mathbf{D}}_{\beta}
\end{aligned}
$$

Using the adaptation laws (21) and (22), Eq.(28) is rewritten as follows:

$$
\begin{aligned}
\dot{L}_{v} \leq \frac{1}{2 \beta} \mathbf{e}^{T}\left(\mathbf{A}^{T} \mathbf{P}+\mathbf{P A}\right) \mathbf{e}+\frac{1}{\beta} \mathbf{e}^{T} \mathbf{P B}\left(\begin{array}{l}
\hat{\mathbf{f}}(\mathbf{x})-\ddot{\mathbf{x}}_{d}+\mathbf{K}^{T} \mathbf{e}+\beta \hat{\mathbf{g}}(\mathbf{x})\left(u_{1}+u_{2}+u_{3}\right)+ \\
+\hat{\mathbf{g}}(\mathbf{x})(\Delta \varphi+\Delta \delta)
\end{array}\right)+ \\
+\frac{1}{\beta} \mathbf{e}^{T} \mathbf{P B D}+\frac{1}{\gamma_{\beta}} \dot{\tilde{\beta}}_{\beta} \tilde{\beta}_{\beta}+\frac{1}{\gamma_{D}} \dot{\mathbf{D}}_{\beta}^{T} \tilde{\mathbf{D}}_{\beta}
\end{aligned}
$$

Using the results in Eq.(20), Eq.(29) is rewritten as follows: 


$$
\begin{aligned}
\dot{L}_{v}=\frac{1}{2 \beta} \mathbf{e}^{T}\left(-\mathbf{Q}-\frac{2}{\rho^{2}} \mathbf{P B B} \mathbf{B}^{T} \mathbf{P}+\beta_{\min } \mathbf{P B R}^{-1} \mathbf{B}^{T} \mathbf{P}\right) \mathbf{e}+\frac{1}{\beta} \mathbf{e}^{T} \mathbf{P B}\left(\begin{array}{l}
\hat{\mathbf{f}}(\mathbf{x})-\ddot{\mathbf{x}}_{d}+\mathbf{K}^{T} \mathbf{e}+ \\
+\beta \hat{\mathbf{g}}(\mathbf{x})\left(u_{1}+u_{2}+u_{3}\right)+ \\
+\hat{\mathbf{g}}(\mathbf{x})(\Delta \varphi+\Delta \delta)
\end{array}\right)+ \\
+\frac{1}{\beta} \mathbf{e}^{T} \mathbf{P B D}+\frac{1}{\gamma_{\beta}} \dot{\tilde{\beta}}_{\beta} \tilde{\beta}_{\beta}+\frac{1}{\gamma_{D}} \dot{\tilde{\mathbf{D}}}_{\beta}^{T} \tilde{\mathbf{D}}_{\beta} \\
\Leftrightarrow \dot{L}_{v} \leq \frac{1}{2 \beta} \mathbf{e}^{T}\left(-\mathbf{Q}-\frac{2}{\rho^{2}} \mathbf{P B B}{ }^{T} \mathbf{P}+\beta_{\min } \mathbf{P B R} \mathbf{R}^{-1} \mathbf{B}^{T} \mathbf{P}\right) \mathbf{e}+\frac{1}{\beta} \mathbf{e}^{T} \mathbf{P B}\left(\hat{\mathbf{f}}(\mathbf{x})-\ddot{\mathbf{x}}_{d}+\mathbf{K}^{T} \mathbf{e}\right)+ \\
+\mathbf{e}^{T} \mathbf{P B} \hat{\mathbf{g}}(\mathbf{x})\left(u_{1}+u_{2}+u_{3}\right)+\left\|\mathbf{e}^{T} \mathbf{P B}\right\|\|\hat{\mathbf{g}}(\mathbf{x})\| \frac{\Delta \varphi+\Delta \delta}{\beta}+ \\
+\frac{1}{\beta} \mathbf{e}^{T} \mathbf{P B D}+\frac{1}{\gamma_{\beta}} \dot{\tilde{\beta}}_{\beta} \tilde{\beta}_{\beta}+\frac{1}{\gamma_{D}} \dot{\tilde{\mathbf{D}}}_{\beta}^{T} \tilde{\mathbf{D}}_{\beta}
\end{aligned}
$$

Using the results in Eqs.(17-19), Eq.(30) is rewritten as follows:

$$
\begin{aligned}
& \Leftrightarrow \dot{L}_{v} \leq \frac{1}{2 \beta} \mathbf{e}^{T}\left(-\mathbf{Q}+\frac{1}{\rho^{2}} \mathbf{P B B}^{T} \mathbf{P}+\beta_{\min } \mathbf{P B R}^{-1} \mathbf{B}^{T} \mathbf{P}\right) \mathbf{e}+\frac{1}{\beta} \mathbf{e}^{T} \mathbf{P B}\left(\hat{\mathbf{f}}(\mathbf{x})-\ddot{\mathbf{x}}_{d}+\mathbf{K}^{T} \mathbf{e}\right)+ \\
& +\mathbf{e}^{T} \mathbf{P B} \hat{\mathbf{g}}(\mathbf{x})\left(-\frac{1}{\beta^{2} \hat{\mathbf{g}}(\mathbf{x})}\left(\hat{\mathbf{f}}(\mathbf{x})-\ddot{\mathbf{x}}_{d}+\mathbf{K}^{T} \mathbf{e}\right)\right)+\mathbf{e}^{T} \mathbf{P B} \hat{\mathbf{g}}(\mathbf{x})\left(-\frac{1}{\beta^{2} \hat{\mathbf{g}}(\mathbf{x})} \mathbf{R}^{-1} \mathbf{B}^{T} \mathbf{P e}\right)+ \\
& +\mathbf{e}^{T} \mathbf{P B} \hat{\mathbf{g}}(\mathbf{x})\left(-\frac{1}{\beta_{\text {min }}}(\Delta \varphi+\Delta \delta) \operatorname{sign}(\hat{\mathbf{g}}(\mathbf{x}))-\frac{1}{\beta^{2} \hat{\mathbf{g}}(\mathbf{x})} \mathbf{D}\right)+ \\
& +\left\|\mathbf{e}^{T} \mathbf{P B}\right\|\|\hat{\mathbf{g}}(\mathbf{x})\| \frac{\Delta \varphi+\Delta \delta}{\beta}+\frac{1}{\beta} \mathbf{e}^{T} \mathbf{P B D}+\frac{1}{\gamma_{\beta}} \dot{\tilde{\beta}}_{\beta} \tilde{\beta}_{\beta}+\frac{1}{\gamma_{D}} \dot{\tilde{\mathbf{D}}}_{\beta}^{T} \tilde{\mathbf{D}}_{\beta} \\
& \Leftrightarrow \dot{L}_{v} \leq \frac{1}{2 \beta} \mathbf{e}^{T}\left(-\mathbf{Q}+\frac{1}{\rho^{2}} \mathbf{P B B}^{T} \mathbf{P}+\beta_{\min } \mathbf{P B R}^{-1} \mathbf{B}^{T} \mathbf{P}\right) \mathbf{e}+\frac{1}{\beta} \mathbf{e}^{T} \mathbf{P B}\left(\hat{\mathbf{f}}(\mathbf{x})-\ddot{\mathbf{x}}_{d}+\mathbf{K}^{T} \mathbf{e}\right)+ \\
& +\mathbf{e}^{T} \mathbf{P B} \hat{\mathbf{g}}(\mathbf{x})\left(-\frac{1}{\beta^{2} \hat{\mathbf{g}}(\mathbf{x})}\left(\hat{\mathbf{f}}(\mathbf{x})-\ddot{\mathbf{x}}_{d}+\mathbf{K}^{T} \mathbf{e}\right)\right)+\mathbf{e}^{T} \mathbf{P B} \hat{\mathbf{g}}(\mathbf{x})\left(-\frac{1}{\beta^{2} \hat{\mathbf{g}}(\mathbf{x})} \mathbf{R}^{-1} \mathbf{B}^{T} \mathbf{P e}\right)+ \\
& +\mathbf{e}^{T} \mathbf{P B} \hat{\mathbf{g}}(\mathbf{x})\left(-\frac{1}{\beta_{\min }}(\Delta \varphi+\Delta \delta) \operatorname{sign}(\hat{\mathbf{g}}(\mathbf{x}))-\frac{1}{\beta^{2} \hat{\mathbf{g}}(\mathbf{x})} \mathbf{D}\right) \\
& +\frac{\Delta \varphi+\Delta \delta}{\beta_{\min }} \operatorname{sign}(\hat{\mathbf{g}}(\mathbf{x})) \mathbf{B}^{T} \mathbf{P e}+\frac{1}{\beta} \mathbf{e}^{T} \mathbf{P B D}+\frac{1}{\gamma_{\beta}} \dot{\tilde{\beta}}_{\beta} \tilde{\beta}_{\beta}+\frac{1}{\gamma_{D}} \dot{\tilde{\mathbf{D}}}_{\beta}^{T} \tilde{\mathbf{D}}_{\beta} \\
& =\frac{1}{2 \beta} \mathbf{e}^{T}\left(-\mathbf{Q}+\frac{1}{\rho^{2}} \mathbf{P B B}^{T} \mathbf{P}+\beta_{\min } \mathbf{P B R}^{-1} \mathbf{B}^{T} \mathbf{P}\right) \mathbf{e}+
\end{aligned}
$$




$$
\begin{aligned}
& +\beta_{\beta} \mathbf{e}^{T} \mathbf{P B}\left(\hat{\mathbf{f}}(\mathbf{x})-\ddot{\mathbf{x}}_{d}+\mathbf{K}^{T} \mathbf{e}\right)-\hat{\beta}_{\beta} \mathbf{e}^{T} \mathbf{P B}\left(\hat{\mathbf{f}}(\mathbf{x})-\ddot{\mathbf{x}}_{d}+\mathbf{K}^{T} \mathbf{e}\right)+\hat{\beta}_{\beta} \mathbf{e}^{T} \mathbf{P B}\left(\hat{\mathbf{f}}(\mathbf{x})-\ddot{\mathbf{x}}_{d}+\mathbf{K}^{T} \mathbf{e}\right)- \\
& -\mathbf{e}^{T} \mathbf{P B} \hat{\mathbf{g}}(\mathbf{x})\left(\frac{\hat{\beta}_{\beta}}{\hat{\mathbf{g}}(\mathbf{x})}\left(\hat{\mathbf{f}}(\mathbf{x})-\ddot{\mathbf{x}}_{d}+\mathbf{K}^{T} \mathbf{e}\right)\right)-\mathbf{e}^{T} \mathbf{P B} \hat{\mathbf{g}}(\mathbf{x})\left(\frac{\hat{\beta}_{\beta}}{\hat{\mathbf{g}}(\mathbf{x})} \mathbf{R}^{-1} \mathbf{B}^{T} \mathbf{P e}\right)+ \\
& -\mathbf{e}^{T} \mathbf{P B} \hat{\mathbf{g}}(\mathbf{x})\left(\frac{1}{\beta_{\min }}(\Delta \varphi+\Delta \delta) \operatorname{sign}(\hat{\mathbf{g}}(\mathbf{x}))\right)-\mathbf{e}^{T} \mathbf{P B} \hat{\mathbf{g}}(\mathbf{x})\left(\frac{1}{\beta \hat{\mathbf{g}}(\mathbf{x})} \hat{\mathbf{D}}_{\beta}\right)+ \\
& +\frac{\Delta \varphi+\Delta \delta}{\beta_{\min }} \operatorname{sign}(\hat{\mathbf{g}}(\mathbf{x})) \mathbf{B}^{T} \mathbf{P e}+\frac{1}{\beta} \mathbf{e}^{T} \mathbf{P B D}+\frac{1}{\gamma_{\beta}} \dot{\tilde{\beta}}_{\beta} \tilde{\beta}_{\beta}+\frac{1}{\gamma_{D}} \dot{\tilde{\mathbf{D}}}_{\beta}^{T} \tilde{\mathbf{D}}_{\beta}
\end{aligned}
$$

Eq.(31) is rewritten as follows:

$$
\begin{aligned}
& \dot{L}_{v} \leq \frac{1}{2 \beta} \mathbf{e}^{T}\left(-\mathbf{Q}+\frac{1}{\rho^{2}} \mathbf{P B B}^{T} \mathbf{P}+\beta_{\min } \mathbf{P B R}^{-1} \mathbf{B}^{T} \mathbf{P}\right) \mathbf{e}+ \\
& +\left(\beta_{\beta}-\hat{\beta}_{\beta}\right) \mathbf{e}^{T} \mathbf{P B}\left(\hat{\mathbf{f}}(\mathbf{x})-\ddot{\mathbf{x}}_{d}+\mathbf{K}^{T} \mathbf{e}\right)+\hat{\beta}_{\beta} \mathbf{e}^{T} \mathbf{P B}\left(\hat{\mathbf{f}}(\mathbf{x})-\ddot{\mathbf{x}}_{d}+\mathbf{K}^{T} \mathbf{e}\right)-\hat{\beta}_{\beta} \mathbf{e}^{T} \mathbf{P B}\left(\hat{\mathbf{f}}(\mathbf{x})-\ddot{\mathbf{x}}_{d}+\mathbf{K}^{T} \mathbf{e}\right)- \\
& -\hat{\beta}_{\beta} \mathbf{e}^{T} \mathbf{P B} \mathbf{R}^{-1} \mathbf{B}^{T} \mathbf{P e}-\frac{1}{\beta_{\text {min }}}(\Delta \varphi+\Delta \delta) \operatorname{sign}(\hat{\mathbf{g}}(\mathbf{x})) \mathbf{B}^{T} \mathbf{P e}-\frac{1}{\beta} \mathbf{e}^{T} \mathbf{P B} \hat{\mathbf{D}}_{\beta}+
\end{aligned}
$$$$
+\frac{\Delta \varphi+\Delta \delta}{\beta_{\min }} \operatorname{sign}(\hat{\mathbf{g}}(\mathbf{x})) \mathbf{B}^{T} \mathbf{P e}+\frac{1}{\beta} \mathbf{e}^{T} \mathbf{P B D}+\frac{1}{\gamma_{\beta}} \dot{\tilde{\beta}}_{\beta} \tilde{\beta}_{\beta}+\frac{1}{\gamma_{D}} \dot{\tilde{\mathbf{D}}}_{\beta}^{T} \tilde{\mathbf{D}}_{\beta}
$$

Eq. (32) is rewritten as follows:

$$
\begin{aligned}
& \dot{L}_{v} \leq \frac{1}{2 \beta} \mathbf{e}^{T}\left(-\mathbf{Q}+\frac{1}{\rho^{2}} \mathbf{P B B}{ }^{T} \mathbf{P}\right) \mathbf{e}+\hat{\beta}_{\beta} \mathbf{e}^{T} \mathbf{P B R} \mathbf{R}^{-1} \mathbf{B}^{T} \mathbf{P e}+\tilde{\beta}_{\beta} \mathbf{e}^{T} \mathbf{P B}\left(\hat{\mathbf{f}}(\mathbf{x})-\ddot{\mathbf{x}}_{d}+\mathbf{K}^{T} \mathbf{e}\right)- \\
& -\hat{\beta}_{\beta} \mathbf{e}^{T} \mathbf{P B R} \mathbf{R}^{-1} \mathbf{B}^{T} \mathbf{P e}-\frac{1}{\beta_{\min }}(\Delta \varphi+\Delta \delta) \operatorname{sign}(\hat{\mathbf{g}}(\mathbf{x})) \mathbf{B}^{T} \mathbf{P e}+\frac{\Delta \varphi+\Delta \delta}{\beta_{\min }} \operatorname{sign}(\hat{\mathbf{g}}(\mathbf{x})) \mathbf{B}^{T} \mathbf{P e}+ \\
& +\frac{1}{\beta} \mathbf{e}^{T} \mathbf{P B}\left(\mathbf{D}-\hat{\mathbf{D}}_{\beta}\right)+\frac{1}{\gamma_{\beta}} \dot{\tilde{\beta}}_{\beta} \tilde{\beta}_{\beta}+\frac{1}{\gamma_{D}} \dot{\tilde{\mathbf{D}}}_{\beta}^{T} \tilde{\mathbf{D}}_{\beta}
\end{aligned}
$$

Using the adaptation laws (23-24), Eq.(33) is rewritten as follows:

$$
\begin{aligned}
& \dot{L}_{v} \leq-\frac{1}{2 \beta} \mathbf{e}^{T} \mathbf{Q e}+\frac{1}{2 \beta \rho^{2}} \mathbf{e}^{T} \mathbf{P B B} \mathbf{B}^{T} \mathbf{P e}=-\frac{1}{2} \beta_{\beta} \mathbf{e}^{T} \mathbf{Q} \mathbf{e}+\frac{1}{2 \rho^{2}} \beta_{\beta} \mathbf{e}^{T} \mathbf{P B B}^{T} \mathbf{P e} \\
& \Leftrightarrow \dot{L}_{v} \leq-\frac{1}{2}\left|\beta_{\beta}\right| \mathbf{e}^{T} \mathbf{Q} \mathbf{e}+\frac{1}{2}\left|\beta_{\beta}\right| \omega^{2}
\end{aligned}
$$


where $\frac{1}{\rho^{2}} \mathbf{e}^{T} \mathbf{P B B}{ }^{T} \mathbf{P e} \leq \omega^{2}$ with $\omega^{2}$ is the upper bound value of $\frac{1}{\rho^{2}} \mathbf{e}^{T} \mathbf{P B B} \mathbf{B}^{T} \mathbf{P e}$. The above equation cannot be used to conclude the system stability. Hence, it needs to be integrated from $t=0$ to $t=T$ :

$L_{v}(0)-L_{v}(T)+\frac{1}{2}\left|\beta_{\beta}\right| \int_{0}^{T} w^{2} d t \geq \frac{1}{2}\left|\beta_{\beta}\right| \int_{0}^{T} \mathbf{e}^{T} \mathbf{Q e} d t$

where, $L_{v}(0)=\frac{1}{2 \beta} \mathbf{e}^{T}(0) \mathbf{P e}(0)+\frac{1}{2 \beta \gamma_{f}} \tilde{\boldsymbol{\Phi}}_{f}^{T}(0) \tilde{\boldsymbol{\Phi}}_{f}(0)+\frac{1}{2 \gamma_{g}} \tilde{\boldsymbol{\Phi}}_{g}^{T}(0) \tilde{\boldsymbol{\Phi}}_{g}(0)+\frac{1}{2 \gamma_{\beta}} \tilde{\beta}_{\beta}^{2}(0)+$.

$+\frac{1}{2 \gamma_{D}} \tilde{\mathbf{D}}_{\beta}^{T}(0) \tilde{\mathbf{D}}_{\beta}(0)+\frac{1}{\gamma_{v}} \dot{\tilde{\mathbf{v}}}^{T}(0) \tilde{\mathbf{v}}(0)$. The value $L_{v}(T)$ is always positive, so Eq. (34) is determined as:

$L_{v}(0)+\frac{1}{2}\left|\beta_{\beta}\right| \int_{0}^{T} w^{2} d t \geq \frac{1}{2}\left|\beta_{\beta}\right| \int_{0}^{T} \mathbf{e}^{T} \mathbf{Q} \mathbf{e} d t \geq 0$

The proof is completed. Figure 2 illustrates the block diagram of the proposed control 1. Firstly, the main controls and the adaptation laws-will use outputs of the fuzzy neural networks model, the modified Riccati-like equation, and the dead-zone band for calculation. The projection algorithm [38] is also applied for the adaptation laws to improve the process. The oOutput of the main control is the input of the plant and the observer. The dĐynamic states of the plant after controlling will be compared with the state variables of the observer, and the results of this comparison are the basis base for the performance evaluation of the proposed control. The eError of this comparison is used as input to updated input of the fuzzy neural networks model and; the adaptation laws. In addition, the states of the plant areis also inputted intoef the fuzzy model. The process is continuously updated until the system states of the plant obtaineding stable convergence.

Remark 1. The update law $\tilde{\beta}_{\beta}$ in (25) will be used to replace the constant parameter $\beta$ in the control inputs $u_{1}, u_{2}, u_{3}$ (in Eqs. (17-19)) to ensure the robustness of the proposed controller. This is a new approach to adapt the gain. Compared to the existing adaptation laws, this modification reduces energy consumption of the control input to minimum value. 


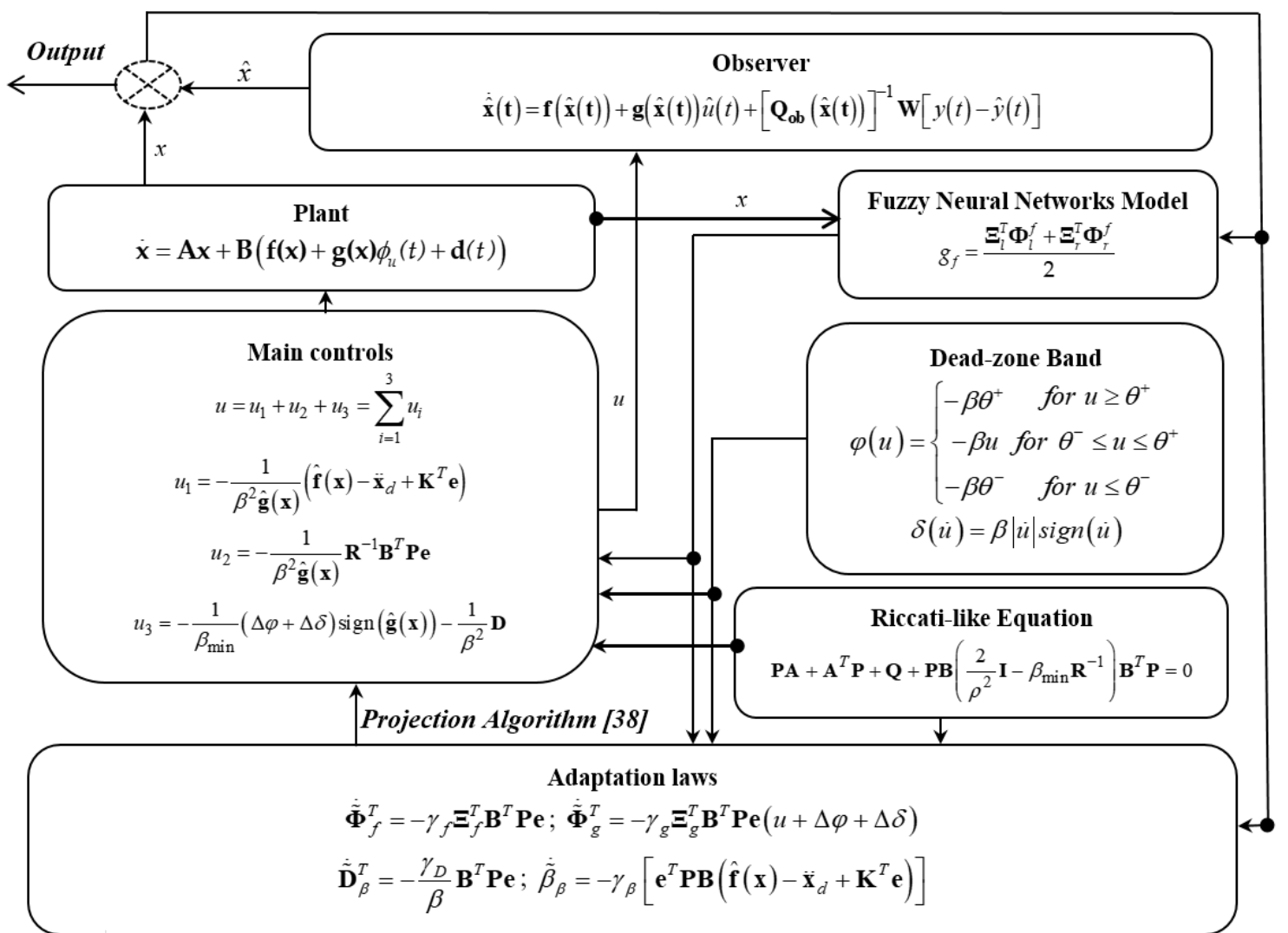

Figure 2. Block diagram of the proposed algorithm 1.

\section{Proposed Control 2.}

In the proposed control 2, full phenomena of the system including dead-zone phenomenon and delay time in actuators presented in Eq. (1) are used. Now, the main control $u$ is determined as follows:

$$
u=u_{1}+u_{2}+u_{3}+u_{4}=\sum_{i=1}^{4} u_{i}
$$

where, $u_{1}$ is the equivalent control, $u_{2}$ is the first robustness control related to the Riccati-like equation, and $u_{3}$ is the second robustness control input, which is used to deal with the dead-zone band, the Riccati-like equation and the minimum approximation error $\mathbf{D}$ of the system, and $u_{4}$ is the third robustness control input, which is used to tackle the effects of the time delay. These controllers are designed as follows:

$$
\begin{aligned}
& u_{1}=-\frac{1}{\beta^{2} \hat{\mathbf{g}}(\mathbf{x})}\left(\hat{\mathbf{f}}(\mathbf{x})-\ddot{\mathbf{x}}_{d}+\mathbf{K}^{T} \mathbf{e}\right) \\
& u_{2}=-\frac{1}{\beta^{2} \hat{\mathbf{g}}(\mathbf{x})} \mathbf{R}^{-1} \mathbf{B}^{T} \mathbf{P e} \\
& u_{3}=-\frac{1}{\beta_{\min 1}}(\Delta \varphi+\Delta \delta) \operatorname{sign}(\hat{\mathbf{g}}(\mathbf{x}))-\frac{1}{\beta^{2} \hat{\mathbf{g}}(\mathbf{x})} \mathbf{D}
\end{aligned}
$$


$u_{4}=-\frac{1}{\beta_{\min 2} \hat{\mathbf{g}}(\mathbf{x})} \hat{\mathbf{v}} \mathbf{B}^{T} \mathbf{P e}$

where, $\Delta \varphi$ is the upper bound value of $\|\varphi(u)\|, \Delta \delta$ is the upper bound value of $\|\delta(\dot{u})\|$. The balance Riccati-like equation is given by

$$
\mathbf{P A}+\mathbf{A}^{T} \mathbf{P}+\mathbf{Q}+\mathbf{P B}\left(\frac{2}{\rho_{1}^{2}} \mathbf{I}-\beta_{\min 1} \mathbf{R}^{-1}\right) \mathbf{B}^{T} \mathbf{P}+\mathbf{P B}\left(\frac{2}{\rho_{2}^{2}} \mathbf{I}-\beta_{\min 2} \mathbf{R}^{-1}\right) \mathbf{B}^{T} \mathbf{P}=0
$$

where, $0<\rho_{1}<1,0<\rho_{2}<1$, I is the identity matrix $(n \times n), \mathbf{Q}=\mathbf{Q}^{T}, \mathbf{R}$ denotes the robust Hinfinity technique gain, $\beta_{\min 1}$ is the parameter related to the dead-zone model, $\beta_{\min 2}$ is the parameter related to the time delay model.

Theorem 2. The stability and robustness of the control system with the dead-zone band and time delay are guaranteed if the control input $u$ is designed as in (36) with its elements shown in Eqs.(37)-(40), and the adaptation laws for these control inputs are chosen as

$$
\begin{aligned}
& \dot{\tilde{\mathbf{\Phi}}}_{f}^{T}=-\gamma_{f} \Xi_{f}^{T} \mathbf{B}^{T} \mathbf{P e} \\
& \dot{\tilde{\mathbf{\Phi}}}_{g}^{T}=-\gamma_{g} \mathbf{\Xi}_{g}^{T} \mathbf{B}^{T} \mathbf{P e}(u+\Delta \varphi+\Delta \delta) \\
& \dot{\tilde{\mathbf{D}}}_{\beta}^{T}=-\frac{\gamma_{D}}{\beta} \mathbf{B}^{T} \mathbf{P e} \\
& \dot{\tilde{\beta}}_{\beta}=-\gamma_{\beta}\left[\mathbf{e}^{T} \mathbf{P B}\left(\hat{\mathbf{f}}(\mathbf{x})-\ddot{\mathbf{x}}_{d}+\mathbf{K}^{T} \mathbf{e}\right)\right] \\
& \dot{\tilde{\mathbf{v}}}^{T}=-\frac{\gamma_{v}}{\beta_{\min 2}}\left(\mathbf{B}^{T} \mathbf{P e}\right)\left(\mathbf{e}^{T} \mathbf{P B}\right)
\end{aligned}
$$

where, $\gamma_{f}, \gamma_{g}, \gamma_{D}, \gamma_{\beta}, \gamma_{v \_}$are chosen constants, $\beta$ is the parameter related to the slope of the deadzone.

Proof: To prove the theorem, a Lyapunov function candidate is proposed as follows:

$$
L_{v}=\frac{1}{2 \beta} \mathbf{e}^{T} \mathbf{P e}+\frac{1}{2 \beta \gamma_{f}} \tilde{\boldsymbol{\Phi}}_{f}^{T} \tilde{\boldsymbol{\Phi}}_{f}+\frac{1}{2 \beta \gamma_{g}} \tilde{\boldsymbol{\Phi}}_{g}^{T} \tilde{\boldsymbol{\Phi}}_{g}+\frac{1}{2 \gamma_{\beta}} \tilde{\beta}_{\beta}^{2}+\frac{1}{2 \gamma_{D}} \tilde{\mathbf{D}}_{\beta}^{T} \tilde{\mathbf{D}}_{\beta}+\frac{1}{2 \gamma_{v}} \tilde{\mathbf{v}}^{T} \tilde{\boldsymbol{v}}
$$

where, $\tilde{\beta}_{\beta}=\beta_{\beta}-\hat{\beta}_{\beta}, \beta_{\beta}=\frac{1}{\beta}, \hat{\beta}_{\beta}=\frac{1}{\beta^{2}}, \hat{\mathbf{D}}_{\beta}=\frac{1}{\beta} \mathbf{D}, \tilde{\mathbf{D}}_{\beta}=\mathbf{D}-\hat{\mathbf{D}}_{\beta}, \boldsymbol{v}=\mathbf{v}-\hat{\mathbf{v}} ; \gamma_{f}, \gamma_{g}, \gamma_{\beta}$, $\gamma_{D}, \gamma_{v}$ are chosen constants.

The derivative of Eq. (47) is obtained as:

$$
\dot{L}_{v}=\frac{1}{2 \beta} \dot{\mathbf{e}}^{T} \mathbf{P e}+\frac{1}{2 \beta} \mathbf{e}^{T} \mathbf{P} \dot{\mathbf{e}}+\frac{1}{\beta \gamma_{f}} \dot{\tilde{\mathbf{\Phi}}}_{f}^{T} \tilde{\boldsymbol{\Phi}}_{f}+\frac{1}{\beta \gamma_{g}} \dot{\tilde{\boldsymbol{\Phi}}}_{g}^{T} \tilde{\boldsymbol{\Phi}}_{g}+\frac{1}{\gamma_{\beta}} \dot{\tilde{\boldsymbol{\beta}}}_{\beta} \tilde{\beta}_{\beta}+\frac{1}{\gamma_{D}} \dot{\tilde{\mathbf{D}}}_{\beta}^{T} \tilde{\mathbf{D}}_{\beta}+\frac{1}{\gamma_{v}} \dot{\tilde{\mathbf{v}}}^{T} \tilde{\mathbf{v}}
$$




$$
\begin{aligned}
\dot{L}_{v}= & \frac{1}{2 \beta} \mathbf{e}^{T}\left(\mathbf{A}^{T} \mathbf{P}+\mathbf{P A}\right) \mathbf{e}+\frac{1}{\beta} \mathbf{e}^{T} \mathbf{P B} \boldsymbol{\Xi}_{f}^{T} \tilde{\boldsymbol{\Phi}}_{f}+\frac{1}{\beta} \mathbf{e}^{T} \mathbf{P B K} \mathbf{K}^{T} \mathbf{e}+\frac{1}{\beta} \mathbf{e}^{T} \mathbf{P B} \boldsymbol{\Xi}_{g}^{T} \tilde{\mathbf{\Phi}}_{g}(\beta u+\Delta \varphi+\Delta \delta)+ \\
& +\frac{1}{\beta} \mathbf{e}^{T} \mathbf{P} \mathbf{B} \hat{\mathbf{f}}(\mathbf{x})+\frac{1}{\beta} \mathbf{e}^{T} \mathbf{P B} \hat{\mathbf{g}}(\mathbf{x})(\beta u+\Delta \varphi+\Delta \delta)+\frac{1}{\beta} \mathbf{e}^{T} \mathbf{P B d}+\frac{1}{\beta} \mathbf{e}^{T} \mathbf{P B D}-\frac{1}{\beta} \mathbf{e}^{T} \mathbf{P B} \ddot{\mathbf{x}}_{d}+ \\
& +\frac{1}{\beta} \mathbf{e}^{T} \mathbf{P B} \boldsymbol{\Lambda}^{T} \mathbf{x}_{\tau}+\frac{1}{\beta \gamma_{f}} \dot{\tilde{\mathbf{\Phi}}}_{f}^{T} \tilde{\mathbf{\Phi}}_{f}+\frac{1}{\beta \gamma_{g}} \dot{\tilde{\mathbf{\Phi}}}_{g}^{T} \tilde{\mathbf{\Phi}}_{g}+\frac{1}{\gamma_{\beta}} \dot{\tilde{\beta}}_{\beta} \tilde{\beta}_{\beta}+\frac{1}{\gamma_{D}} \dot{\tilde{\mathbf{D}}}_{\beta}^{T} \tilde{\mathbf{D}}_{\beta}+\frac{1}{\gamma_{v}} \dot{\tilde{\mathbf{v}}}^{T} \tilde{\mathbf{v}}
\end{aligned}
$$

Using the adaptation laws (42) and (43), Eq.(49) is rewritten as follows:

$$
\begin{gathered}
\dot{L}_{v} \leq \frac{1}{2 \beta} \mathbf{e}^{T}\left(\mathbf{A}^{T} \mathbf{P}+\mathbf{P A}\right) \mathbf{e}+\frac{1}{\beta} \mathbf{e}^{T} \mathbf{P B}\left(\begin{array}{l}
\hat{\mathbf{f}}(\mathbf{x})-\ddot{\mathbf{x}}_{d}+\mathbf{K}^{T} \mathbf{e}+\beta \hat{\mathbf{g}}(\mathbf{x})\left(u_{1}+u_{2}+u_{3}+u_{4}\right)+ \\
+\hat{\mathbf{g}}(\mathbf{x})(\Delta \varphi+\Delta \delta)
\end{array}\right)+ \\
+\frac{1}{\beta} \mathbf{e}^{T} \mathbf{P B D}+\frac{1}{\beta} \mathbf{e}^{T} \mathbf{P B} \boldsymbol{\Lambda}^{T} \mathbf{x}_{\tau}+\frac{1}{\gamma_{\beta}} \dot{\tilde{\beta}}_{\beta} \tilde{\beta}_{\beta}+\frac{1}{\gamma_{D}} \dot{\tilde{\mathbf{D}}}_{\beta}^{T} \tilde{\mathbf{D}}_{\beta}+\frac{1}{\gamma_{v}} \dot{\tilde{\mathbf{v}}}^{T} \tilde{\mathbf{v}}
\end{gathered}
$$

Using Eq.(41), Eq.(50) is rewritten as follows:

$$
\begin{aligned}
& \dot{L}_{v}=\frac{1}{2 \beta} \mathbf{e}^{T}\left(\begin{array}{l}
-\mathbf{Q}-\frac{2}{\rho_{1}^{2}} \mathbf{P B B} \mathbf{B}^{T} \mathbf{P}+\beta_{\min 1} \mathbf{P B R}^{-1} \mathbf{B}^{T} \mathbf{P}- \\
-\frac{2}{\rho_{2}^{2}} \mathbf{P B B}^{T} \mathbf{P}+\beta_{\min 2} \mathbf{P B R}^{-1} \mathbf{B}^{T} \mathbf{P}
\end{array}\right) \mathbf{e}+\frac{1}{\beta} \mathbf{e}^{T} \mathbf{P B}\left(\begin{array}{l}
\hat{\mathbf{f}}(\mathbf{x})-\ddot{\mathbf{x}}_{d}+\mathbf{K}^{T} \mathbf{e}+ \\
+\beta \hat{\mathbf{g}}(\mathbf{x})\left(u_{1}+u_{2}+u_{3}+u_{4}\right)+ \\
+\hat{\mathbf{g}}(\mathbf{x})(\Delta \varphi+\Delta \delta)
\end{array}\right)+ \\
& +\frac{1}{\beta} \mathbf{e}^{T} \mathbf{P B D}+\frac{1}{\beta} \mathbf{e}^{T} \mathbf{P B} \boldsymbol{\Lambda}^{T} \mathbf{x}_{\tau}+\frac{1}{\gamma_{\beta}} \dot{\tilde{\beta}}_{\beta} \tilde{\beta}_{\beta}+\frac{1}{\gamma_{D}} \dot{\tilde{\mathbf{D}}}_{\beta}^{T} \tilde{\mathbf{D}}_{\beta}+\frac{1}{\gamma_{v}} \dot{\tilde{\mathbf{v}}}^{T} \tilde{\mathbf{v}} \\
& \Leftrightarrow \dot{L}_{v} \leq \frac{1}{2 \beta} \mathbf{e}^{T}\left(\begin{array}{l}
-\mathbf{Q}-\frac{2}{\rho_{1}^{2}} \mathbf{P B B}^{T} \mathbf{P}+\beta_{\min 1} \mathbf{P B R}^{-1} \mathbf{B}^{T} \mathbf{P}- \\
-\frac{2}{\rho_{2}^{2}} \mathbf{P B B}^{T} \mathbf{P}+\beta_{\min 2} \mathbf{P B R}^{-1} \mathbf{B}^{T} \mathbf{P}
\end{array}\right) \mathbf{e}+\frac{1}{\beta} \mathbf{e}^{T} \mathbf{P B}\left(\hat{\mathbf{f}}(\mathbf{x})-\ddot{\mathbf{x}}_{d}+\mathbf{K}^{T} \mathbf{e}\right)+ \\
& +\mathbf{e}^{T} \mathbf{P B} \hat{\mathbf{g}}(\mathbf{x})\left(u_{1}+u_{2}+u_{3}+u_{4}\right)+\left\|\mathbf{e}^{T} \mathbf{P B}\right\|\|\hat{\mathbf{g}}(\mathbf{x})\| \frac{\Delta \varphi+\Delta \delta}{\beta}+ \\
& +\frac{1}{\beta} \mathbf{e}^{T} \mathbf{P B D}+\frac{1}{\beta} \mathbf{e}^{T} \mathbf{P B} \boldsymbol{\Lambda}^{T} \mathbf{x}_{\tau}+\frac{1}{\gamma_{\beta}} \dot{\tilde{\beta}}_{\beta} \tilde{\beta}_{\beta}+\frac{1}{\gamma_{D}} \dot{\tilde{\mathbf{D}}}_{\beta}^{T} \tilde{\mathbf{D}}_{\beta}+\frac{1}{\gamma_{v}} \dot{\tilde{\mathbf{v}}}^{T} \tilde{\mathbf{v}}
\end{aligned}
$$

Using Eqs.(37)-(40), Eq.(51) is rewritten as follows:

$$
\Leftrightarrow \dot{L}_{v} \leq \frac{1}{2 \beta} \mathbf{e}^{T}\left(\begin{array}{l}
-\mathbf{Q}+\frac{1}{\rho_{1}^{2}} \mathbf{P B B}^{T} \mathbf{P}+\beta_{\text {min } 1} \mathbf{P B R}^{-1} \mathbf{B}^{T} \mathbf{P}+ \\
+\frac{1}{\rho_{2}^{2}} \mathbf{P B B}^{T} \mathbf{P}+\beta_{\text {min } 2} \mathbf{P B R}^{-1} \mathbf{B}^{T} \mathbf{P}
\end{array}\right) \mathbf{e}+\frac{1}{\beta} \mathbf{e}^{T} \mathbf{P B}\left(\hat{\mathbf{f}}(\mathbf{x})-\ddot{\mathbf{x}}_{d}+\mathbf{K}^{T} \mathbf{e}\right)+
$$




$$
\begin{aligned}
& +\mathbf{e}^{T} \mathbf{P B} \hat{\mathbf{g}}(\mathbf{x})\left(-\frac{1}{\beta^{2} \hat{\mathbf{g}}(\mathbf{x})}\left(\hat{\mathbf{f}}(\mathbf{x})-\ddot{\mathbf{x}}_{d}+\mathbf{K}^{T} \mathbf{e}\right)\right)+\mathbf{e}^{T} \mathbf{P B} \hat{\mathbf{g}}(\mathbf{x})\left(-\frac{1}{\beta^{2} \hat{\mathbf{g}}(\mathbf{x})} \mathbf{R}^{-1} \mathbf{B}^{T} \mathbf{P e}\right)+ \\
& +\mathbf{e}^{T} \mathbf{P B} \hat{\mathbf{g}}(\mathbf{x})\left(-\frac{1}{\beta_{\min 1}}(\Delta \varphi+\Delta \delta) \operatorname{sign}(\hat{\mathbf{g}}(\mathbf{x}))-\frac{1}{\beta^{2} \hat{\mathbf{g}}(\mathbf{x})} \mathbf{D}\right)+\mathbf{e}^{T} \mathbf{P B} \hat{\mathbf{g}}(\mathbf{x})\left(-\frac{1}{\beta_{\min 2} \hat{\mathbf{g}}(\mathbf{x})} \hat{\mathbf{v}} \mathbf{B}^{T} \mathbf{P e}\right)+ \\
& +\left\|\mathbf{e}^{T} \mathbf{P B}\right\|\|\hat{\mathbf{g}}(\mathbf{x})\| \frac{\Delta \varphi+\Delta \delta}{\beta}+\frac{1}{\beta} \mathbf{e}^{T} \mathbf{P B D}+\frac{1}{\beta} \mathbf{e}^{T} \mathbf{P B} \Lambda^{T} \mathbf{x}_{\tau}+\frac{1}{\gamma_{\beta}} \dot{\tilde{\beta}}_{\beta} \tilde{\beta}_{\beta}+\frac{1}{\gamma_{D}} \dot{\tilde{\mathbf{D}}}_{\beta}^{T} \tilde{\mathbf{D}}_{\beta}+\frac{1}{\gamma_{v}} \dot{\tilde{\mathbf{v}}}^{T} \tilde{\mathbf{v}} \\
& \Leftrightarrow \dot{L}_{v} \leq \frac{1}{2 \beta} \mathbf{e}^{T}\left(\begin{array}{l}
-\mathbf{Q}+\frac{1}{\rho_{1}^{2}} \mathbf{P B B} \mathbf{B}^{T} \mathbf{P}+\beta_{\min 1} \mathbf{P B R}^{-1} \mathbf{B}^{T} \mathbf{P}+ \\
+\frac{1}{\rho_{2}^{2}} \mathbf{P B B}^{T} \mathbf{P}+\beta_{\min 2} \mathbf{P B R}^{-1} \mathbf{B}^{T} \mathbf{P}
\end{array}\right) \mathbf{e}+\frac{1}{\beta} \mathbf{e}^{T} \mathbf{P B}\left(\hat{\mathbf{f}}(\mathbf{x})-\ddot{\mathbf{x}}_{d}+\mathbf{K}^{T} \mathbf{e}\right)+ \\
& +\mathbf{e}^{T} \mathbf{P B} \hat{\mathbf{g}}(\mathbf{x})\left(-\frac{1}{\beta^{2} \hat{\mathbf{g}}(\mathbf{x})}\left(\hat{\mathbf{f}}(\mathbf{x})-\ddot{\mathbf{x}}_{d}+\mathbf{K}^{T} \mathbf{e}\right)\right)+\mathbf{e}^{T} \mathbf{P B} \hat{\mathbf{g}}(\mathbf{x})\left(-\frac{1}{\beta^{2} \hat{\mathbf{g}}(\mathbf{x})} \mathbf{R}^{-1} \mathbf{B}^{T} \mathbf{P e}\right)+ \\
& +\mathbf{e}^{T} \mathbf{P B} \hat{\mathbf{g}}(\mathbf{x})\left(-\frac{1}{\beta_{\min 1}}(\Delta \varphi+\Delta \delta) \operatorname{sign}(\hat{\mathbf{g}}(\mathbf{x}))-\frac{1}{\beta^{2} \hat{\mathbf{g}}(\mathbf{x})} \mathbf{D}\right)+\mathbf{e}^{T} \mathbf{P B} \hat{\mathbf{g}}(\mathbf{x})\left(-\frac{1}{\beta_{\min 2} \hat{\mathbf{g}}(\mathbf{x})} \hat{\mathbf{v}} \mathbf{B}^{T} \mathbf{P e}\right)+ \\
& +\frac{\Delta \varphi+\Delta \delta}{\beta_{\min 1}} \operatorname{sign}(\hat{\mathbf{g}}(\mathbf{x})) \mathbf{B}^{T} \mathbf{P e}+\frac{1}{\beta} \mathbf{e}^{T} \mathbf{P B D}+\frac{1}{\beta} \mathbf{e}^{T} \mathbf{P B} \mathbf{\Lambda}^{T} \mathbf{x}_{\tau}+\frac{1}{\gamma_{\beta}} \dot{\tilde{\beta}}_{\beta} \tilde{\beta}_{\beta}+\frac{1}{\gamma_{D}} \dot{\tilde{\mathbf{D}}}_{\beta}^{T} \tilde{\mathbf{D}}_{\beta}+\frac{1}{\gamma_{v}} \dot{\tilde{\mathbf{v}}}^{T} \tilde{\mathbf{v}} \\
& =\frac{1}{2 \beta} \mathbf{e}^{T}\left(-\mathbf{Q}+\left(\frac{1}{\rho_{1}^{2}}+\frac{1}{\rho_{2}^{2}}\right) \mathbf{P B B}^{T} \mathbf{P}+\left(\beta_{\min 1}+\beta_{\min 2}\right) \mathbf{P B R}^{-1} \mathbf{B}^{T} \mathbf{P}\right) \mathbf{e}+ \\
& +\beta_{\beta} \mathbf{e}^{T} \mathbf{P B}\left(\hat{\mathbf{f}}(\mathbf{x})-\ddot{\mathbf{x}}_{d}+\mathbf{K}^{T} \mathbf{e}\right)-\hat{\beta}_{\beta} \mathbf{e}^{T} \mathbf{P B}\left(\hat{\mathbf{f}}(\mathbf{x})-\ddot{\mathbf{x}}_{d}+\mathbf{K}^{T} \mathbf{e}\right)+\hat{\beta}_{\beta} \mathbf{e}^{T} \mathbf{P B}\left(\hat{\mathbf{f}}(\mathbf{x})-\ddot{\mathbf{x}}_{d}+\mathbf{K}^{T} \mathbf{e}\right) \\
& -\mathbf{e}^{T} \mathbf{P B} \hat{\mathbf{g}}(\mathbf{x})\left(\frac{\hat{\beta}_{\beta}}{\hat{\mathbf{g}}(\mathbf{x})}\left(\hat{\mathbf{f}}(\mathbf{x})-\ddot{\mathbf{x}}_{d}+\mathbf{K}^{T} \mathbf{e}\right)\right)-\mathbf{e}^{T} \mathbf{P B} \hat{\mathbf{g}}(\mathbf{x})\left(\frac{\hat{\beta}_{\beta}}{\hat{\mathbf{g}}(\mathbf{x})} \mathbf{R}^{-1} \mathbf{B}^{T} \mathbf{P e}\right)+ \\
& -\mathbf{e}^{T} \mathbf{P B} \hat{\mathbf{g}}(\mathbf{x})\left(\frac{1}{\beta_{\min 1}}(\Delta \varphi+\Delta \delta) \operatorname{sign}(\hat{\mathbf{g}}(\mathbf{x}))\right)-\mathbf{e}^{T} \mathbf{P B} \hat{\mathbf{g}}(\mathbf{x})\left(\frac{1}{\beta \hat{\mathbf{g}}(\mathbf{x})} \hat{\mathbf{D}}_{\beta}\right)-\mathbf{e}^{T} \mathbf{P B} \hat{\mathbf{g}}(\mathbf{x})\left(\frac{1}{\beta_{\min 2} \hat{\mathbf{g}}(\mathbf{x})} \hat{\mathbf{v}} \mathbf{B}^{T} \mathbf{P e}\right)+ \\
& +\frac{\Delta \varphi+\Delta \delta}{\beta_{\min 1}} \operatorname{sign}(\hat{\mathbf{g}}(\mathbf{x})) \mathbf{B}^{T} \mathbf{P e}+\frac{1}{\beta} \mathbf{e}^{T} \mathbf{P B D}+\frac{1}{\beta} \mathbf{e}^{T} \mathbf{P B} \boldsymbol{\Lambda}^{T} \mathbf{x}_{\tau}+\frac{1}{\gamma_{\beta}} \dot{\tilde{\beta}}_{\beta} \tilde{\beta}_{\beta}+\frac{1}{\gamma_{D}} \dot{\tilde{\mathbf{D}}}_{\beta}^{T} \tilde{\mathbf{D}}_{\beta}+\frac{1}{\gamma_{v}} \dot{\tilde{\mathbf{v}}}^{T} \tilde{\mathbf{v}}
\end{aligned}
$$

Now, Eq.(52) is rewritten as follows:

$$
\begin{aligned}
& \dot{L}_{v} \leq \frac{1}{2 \beta} \mathbf{e}^{T}\left(-\mathbf{Q}+\frac{1}{\rho^{2}} \mathbf{P B B}^{T} \mathbf{P}+\left(\beta_{\min 1}+\beta_{\min 2}\right) \mathbf{P B R}^{-1} \mathbf{B}^{T} \mathbf{P}\right) \mathbf{e}+ \\
& +\left(\beta_{\beta}-\hat{\beta}_{\beta}\right) \mathbf{e}^{T} \mathbf{P B}\left(\hat{\mathbf{f}}(\mathbf{x})-\ddot{\mathbf{x}}_{d}+\mathbf{K}^{T} \mathbf{e}\right)+\hat{\beta}_{\beta} \mathbf{e}^{T} \mathbf{P B}\left(\hat{\mathbf{f}}(\mathbf{x})-\ddot{\mathbf{x}}_{d}+\mathbf{K}^{T} \mathbf{e}\right)-\hat{\beta}_{\beta} \mathbf{e}^{T} \mathbf{P B}\left(\hat{\mathbf{f}}(\mathbf{x})-\ddot{\mathbf{x}}_{d}+\mathbf{K}^{T} \mathbf{e}\right)-
\end{aligned}
$$


$-\hat{\beta}_{\beta} \mathbf{e}^{T} \mathbf{P B R} \mathbf{R}^{-1} \mathbf{B}^{T} \mathbf{P e}-\frac{1}{\beta_{\min 1}}(\Delta \varphi+\Delta \delta) \operatorname{sign}(\hat{\mathbf{g}}(\mathbf{x})) \mathbf{B}^{T} \mathbf{P e}-\frac{1}{\beta} \mathbf{e}^{T} \mathbf{P B} \hat{\mathbf{D}}_{\beta}-\frac{1}{\beta_{\min 2}} \hat{\mathbf{v}}^{T} \mathbf{P e e}^{T} \mathbf{P B}+$ $+\frac{\Delta \varphi+\Delta \delta}{\beta_{\min 1}} \operatorname{sign}(\hat{\mathbf{g}}(\mathbf{x})) \mathbf{B}^{T} \mathbf{P e}+\frac{1}{\beta} \mathbf{e}^{T} \mathbf{P B D}+\frac{1}{\beta_{\min 2}} \mathbf{v} \mathbf{B}^{T} \mathbf{P} \mathbf{e}^{T} \mathbf{P B}+\frac{1}{\gamma_{\beta}} \dot{\tilde{\beta}}_{\beta} \tilde{\beta}_{\beta}+\frac{1}{\gamma_{D}} \dot{\tilde{\mathbf{D}}}_{\beta}^{T} \tilde{\mathbf{D}}_{\beta}+\frac{1}{\gamma_{v}} \dot{\tilde{\mathbf{v}}}^{T} \tilde{\mathbf{v}}$

where, $\frac{1}{\rho^{2}}=\frac{1}{\rho_{1}^{2}}+\frac{1}{\rho_{2}^{2}}, 0<\rho<1$.

Thus, Eq.(53) is rewritten as follows:

$\dot{L}_{v} \leq \frac{1}{2 \beta} \mathbf{e}^{T}\left(-\mathbf{Q}+\frac{1}{\rho^{2}} \mathbf{P B B}^{T} \mathbf{P}\right) \mathbf{e}+\hat{\beta}_{\beta} \mathbf{e}^{T} \mathbf{P B R} \mathbf{R}^{-1} \mathbf{B}^{T} \mathbf{P e}+\tilde{\beta}_{\beta} \mathbf{e}^{T} \mathbf{P B}\left(\hat{\mathbf{f}}(\mathbf{x})-\ddot{\mathbf{x}}_{d}+\mathbf{K}^{T} \mathbf{e}\right)+$

$-\hat{\beta}_{\beta} \mathbf{e}^{T} \mathbf{P B R} \mathbf{R}^{-1} \mathbf{B}^{T} \mathbf{P e}-\frac{1}{\beta_{\min 1}}(\Delta \varphi+\Delta \delta) \operatorname{sign}(\hat{\mathbf{g}}(\mathbf{x})) \mathbf{B}^{T} \mathbf{P e}+\frac{\Delta \varphi+\Delta \delta}{\beta_{\min 1}} \operatorname{sign}(\hat{\mathbf{g}}(\mathbf{x})) \mathbf{B}^{T} \mathbf{P e}$

$+\frac{1}{\beta} \mathbf{e}^{T} \mathbf{P B}\left(\mathbf{D}-\hat{\mathbf{D}}_{\beta}\right)+\frac{1}{\beta_{\min 2}}(\boldsymbol{v}-\hat{\mathbf{v}}) \mathbf{B}^{T} \mathbf{P} \mathbf{e e}^{T} \mathbf{P B}+\frac{1}{\gamma_{\beta}} \dot{\tilde{\beta}}_{\beta} \tilde{\beta}_{\beta}+\frac{1}{\gamma_{D}} \dot{\tilde{\mathbf{D}}}_{\beta}^{T} \tilde{\mathbf{D}}_{\beta}+\frac{1}{\gamma_{v}} \dot{\tilde{\mathbf{v}}}^{T} \tilde{\mathbf{v}}$

Using the adaptation laws (44)-(46), Eq.(54) is rewritten as follows:

$\dot{L}_{v} \leq-\frac{1}{2 \beta} \mathbf{e}^{T} \mathbf{Q e}+\frac{1}{2 \beta \rho^{2}} \mathbf{e}^{T} \mathbf{P B B}{ }^{T} \mathbf{P e}=-\frac{1}{2} \beta_{\beta} \mathbf{e}^{T} \mathbf{Q e}+\frac{1}{2 \rho^{2}} \beta_{\beta} \mathbf{e}^{T} \mathbf{P B B} \mathbf{B}^{T} \mathbf{P e}$

$\Leftrightarrow \dot{L}_{v} \leq-\frac{1}{2}\left|\beta_{\beta}\right| \mathbf{e}^{T} \mathbf{Q} \mathbf{e}+\frac{1}{2}\left|\beta_{\beta}\right| \omega^{2}$

where, $\frac{1}{\rho^{2}} \mathbf{e}^{T} \mathbf{P B B}{ }^{T} \mathbf{P e} \leq \omega^{2}$ with $\omega^{2}$ is the upper bound value of $\frac{1}{\rho^{2}} \mathbf{e}^{T} \mathbf{P B B}{ }^{T} \mathbf{P e}$. The above equation cannot be used to conclude the system stability. Hence, it needs to be integrated from $t=0$ to $t=T$ :

$L_{v}(0)-L_{v}(T)+\frac{1}{2}\left|\beta_{\beta}\right| \int_{0}^{T} w^{2} d t \geq \frac{1}{2}\left|\beta_{\beta}\right| \int_{0}^{T} \mathbf{e}^{T} \mathbf{Q e} d t$

where, $L_{v}(0)=\frac{1}{2 \beta} \mathbf{e}^{T}(0) \mathbf{P e}(0)+\frac{1}{2 \beta \gamma_{f}} \tilde{\boldsymbol{\Phi}}_{f}^{T}(0) \tilde{\boldsymbol{\Phi}}_{f}(0)+\frac{1}{2 \gamma_{g}} \tilde{\boldsymbol{\Phi}}_{g}^{T}(0) \tilde{\boldsymbol{\Phi}}_{g}(0)+\frac{1}{2 \gamma_{\beta}} \tilde{\boldsymbol{\beta}}_{\beta}^{2}(0)+$.

$+\frac{1}{2 \gamma_{D}} \tilde{\mathbf{D}}_{\beta}^{T}(0) \tilde{\mathbf{D}}_{\beta}(0)+\frac{1}{\gamma_{v}} \dot{\tilde{\mathbf{v}}}^{T}(0) \tilde{\mathbf{v}}(0)$. The value $L_{v}(T)$ is always positive, and thus Eq. (55) is

determined as: 


$$
L_{v}(0)+\frac{1}{2}\left|\beta_{\beta}\right| \int_{0}^{T} w^{2} d t \geq \frac{1}{2}\left|\beta_{\beta}\right| \int_{0}^{T} \mathbf{e}^{T} \mathbf{Q e} d t \geq 0
$$

The proof is now completed. Figure 3 illustrates the block diagram of the proposed control 2. The control process is similar to the Figure 2. However, the $\underline{a}$ dead-zone band-delayed model and $\underline{a}$ new modified Riccati-like equation are added for calculation.

Remark 2. As a similar way as Remark 1, the proposed adaptive law in (45) helps to reduce the energy consumption in control inputs.

Remark 3. In [18], the control process usingapproach used fuzzy model to handle thefor deadzone. However, this approach requires higher computational burden eonsumed more time for ealeulation because of the overlap of the iteration parameters. The Nussbaum-type function was used tofor supporting this process. However, this application-was affects-to the system stabilityeontrel and the-delayed time delay issues was not consideredselved thoroughly.

Remark 4. In [19], the model of neural networks was applied for the dead-zone problem.

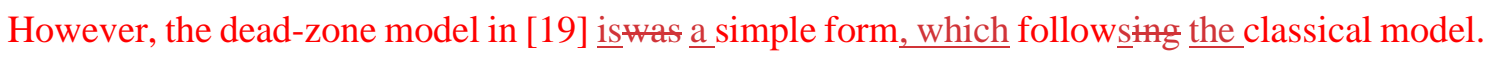
In addition, tThe delayed timetime delay issue was not solved in [19].

Remark 5. In [20], the dead-zone model was similar to the model in [19]. The delayed timetime delay of actuator was not mentioned considered in [19]. Hence, the proposed controllers in $[19,20]$ were notcould not obtain the required precision in controllevel for a complicated complex system. The delay and dead-zone of oil in hydraulic system [20] was impacted by pressure in valve and the pipe line.

Remark 6. In [21], the dead-zone model was still formed like the classical model. The inverse model was used with the adaptation laws for control. This inverse model wais the main disadvantage of the control system [21], which increases $\underline{\text { d }}$ the time of calculation time and the convergence of the desired system error. The delayed time was not taken into consideration added in [21].

Remark 7. In [22], the classical model of dead-zone was still applied. The prescribed performance was used for eontrol-defining the convergence of the error. However, the obtained results were similarlike the results in [21] with the properties-as mentioned-discussed above.

Remark 8. In [23], the dead-zone model was developed based on the classical model. The time delay issue was also mentioned-discussed in the model [23]. However, this approach preserves the disadvantages as that of e disadvantages of the proposed model was remain as shown in the classical model. This will be pointed out in the next section, and the control [23] is aswill be also compared eontrol-with the proposed control in this study.

\section{Simulation and Discussion}

\subsection{Model of Simulation}


In this work, the vehicle seat suspension system with MR damper studied in [13] is adopted to demonstrate the effectiveness of the proposed controller. The mechanical model of the system is shown in Figure 4. The governing equations of the system are derived as follows:

$$
\begin{aligned}
& m_{s} \ddot{x}_{s}=-k_{s}\left(x_{s}-x_{0}\right)-c_{s}\left(\dot{x}_{s}-\dot{x}_{0}\right)+k_{1}\left(x_{1}-x_{s}\right)+c_{1}\left(\dot{x}_{1}-\dot{x}_{s}\right)+F_{M R} \\
& m_{1} \ddot{x}_{1}=-k_{1}\left(x_{1}-x_{s}\right)+c_{1}\left(\dot{x}_{1}-\dot{x}_{s}\right)
\end{aligned}
$$

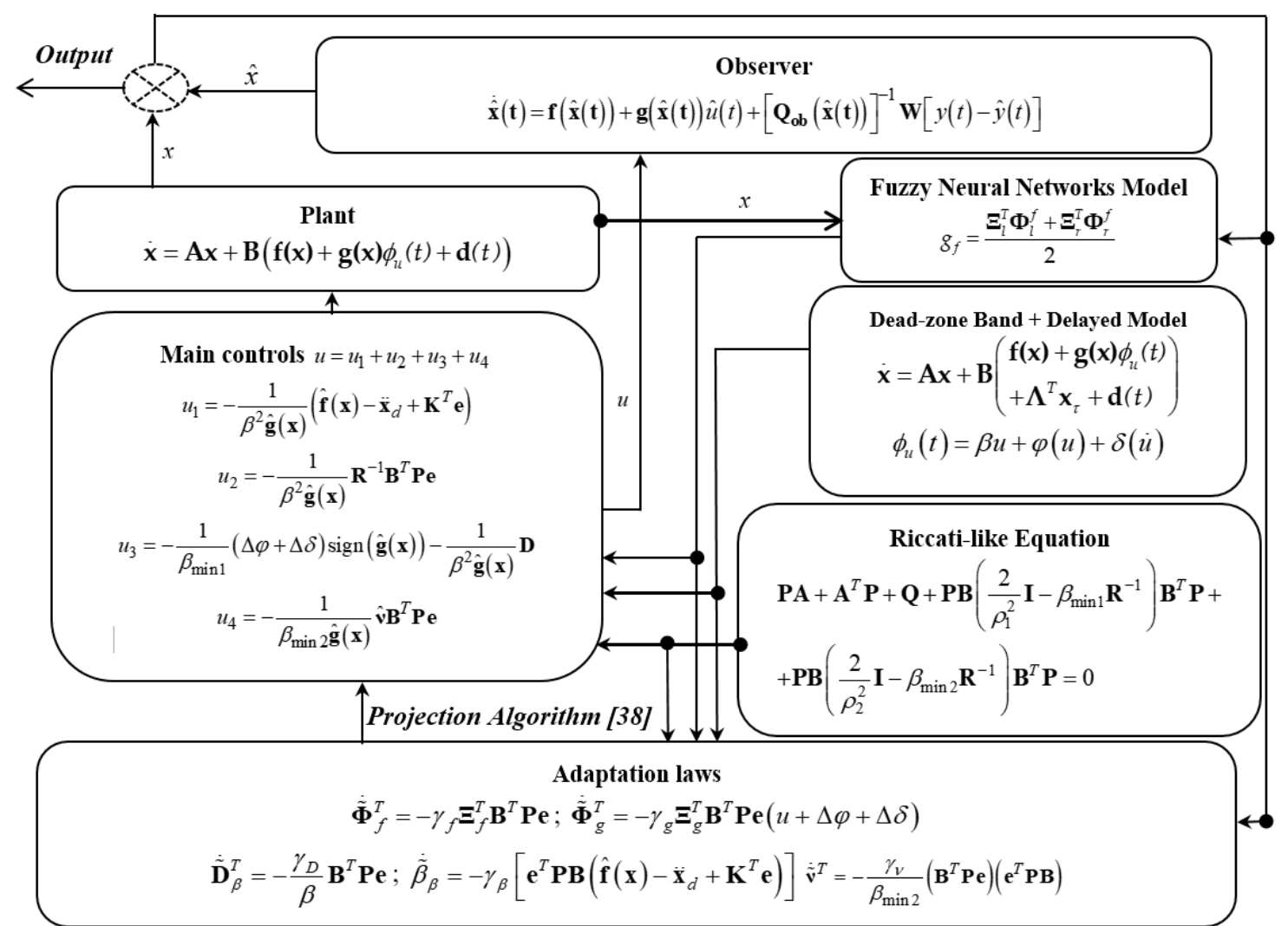

Figure 3. Block diagram of the proposed algorithm 2.

The above equations can be rewritten using the state variables as follows:

$$
\begin{gathered}
\dot{x}_{11}=\dot{x}_{s}=x_{22} \\
\dot{x}_{22}=f_{11}\left(x_{11}, x_{22}, x_{33}, x_{44}\right)+g_{11}\left(x_{11}, x_{22}, x_{33}, x_{44}\right) u \\
\dot{x}_{33}=\dot{x}_{1}=x_{44} \\
\dot{x}_{44}=f_{22}\left(x_{11}, x_{22}, x_{33}, x_{44}\right)
\end{gathered}
$$

where,

$$
\begin{aligned}
& f_{11}\left(x_{11}, x_{22}, x_{33}, x_{44}\right)=-\frac{k_{s}}{m_{s}}\left(x_{11}-x_{0}\right)-\frac{c_{s}}{m_{s}}\left(x_{22}-\dot{x}_{0}\right)+\frac{k_{1}}{m_{s}}\left(x_{33}-x_{11}\right)+\frac{c_{1}}{m_{s}}\left(x_{44}-x_{22}\right) \\
& g_{11}\left(x_{11}, x_{22}, x_{33}, x_{44}\right)=\frac{1}{m_{s}}, u=F_{M R}, f_{22}\left(x_{11}, x_{22}, x_{33}, x_{44}\right)=-\frac{k_{1}}{m_{1}}\left(x_{33}-x_{11}\right)-\frac{c_{1}}{m_{1}}\left(x_{44}-x_{22}\right) .
\end{aligned}
$$


It is notice that $x_{11}, x_{22}, x_{33}, x_{44}$ are variables related to $x_{s}$ and $x_{1}$ of the system (57)-(58).

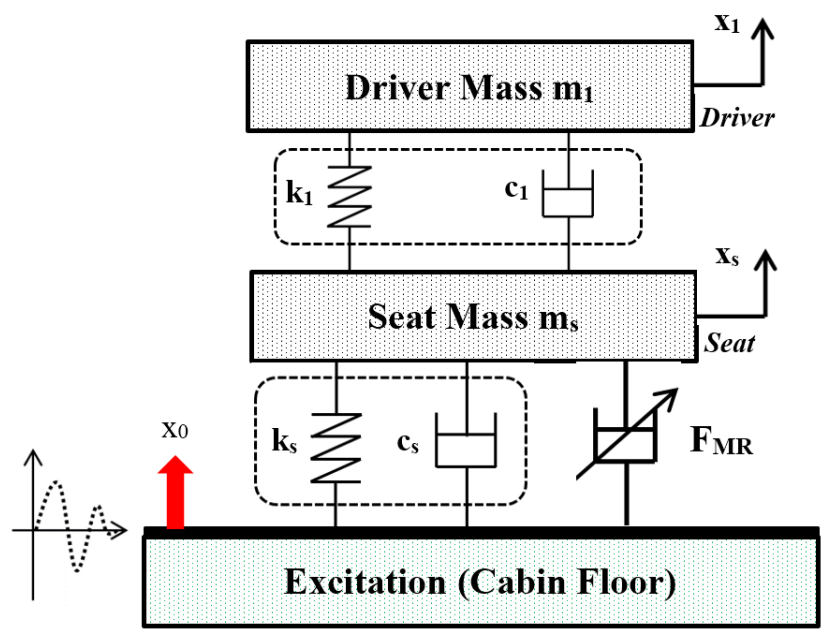

Figure 4. Mechanical model of the seat suspension.

From Figure 1, the dead-zone for MR damper or generally MR device can be represented in Figure $5(\mathrm{a}, \mathrm{b}, \mathrm{c})$. It is remarked that there is also another phenomenon namely hysteresis in MR device. Normally, hysteresis phenomenon exists in the model of MR device. This phenomenon is explained as a nondegenerate input-output closed curve, which defines the frequency of excitation towards a DC signals [37], or a discontinuous jump in the force-velocity response [31]. The hysteresis phenomenon leads to some problems such as high tracking error, unwanted harmonics, and instability of the system. The difference between the dead-zone and hysteresis is that the value of the output will be zero for dead-zone, but a mean value for hysteresis. The disadvantages

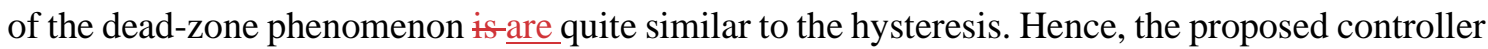
for dead-zone problem is also true for the hysteresis issteproblem.

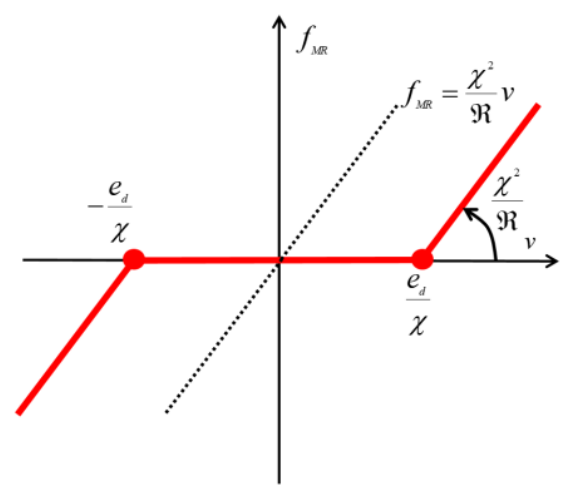

(a)

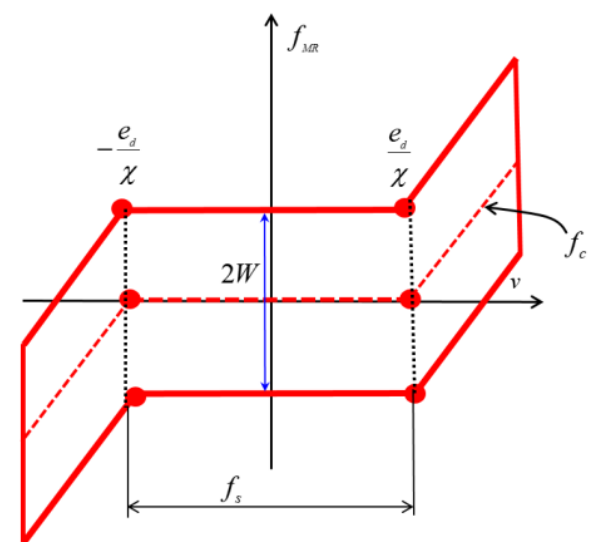

(b) 
Figure 5. Parameters of damping force for dead-zone model: (a) Fixed-frame model, (b) Realframe model

From Eq.(5), the main control function shown in Figure 5(a) can be written as follows:

$\phi\left(f_{M R}\right)=\beta f_{M R}+\varphi\left(f_{M R}\right)+\delta\left(\dot{f}_{M R}\right)$

where, $f_{M R}$ is the theoretical damping force of the damper. Using similar definitions used in [32], the parameters of Eq.(60) are defined as:

$f_{M R}=\frac{\chi^{2}}{\mathfrak{R}} v, \dot{f}_{M R}=\frac{\chi^{2}}{\mathfrak{R}} \dot{v}$

where, $\chi(\mathrm{V} . \mathrm{s} / \mathrm{m})$ is the actuation constant related to the viscous coefficient, $\Re$ is the resistance of the circuit of the damper $(\Omega), v(\mathrm{~m} / \mathrm{s})$ is the velocity of the damper. The function $\varphi\left(f_{M R}\right)$ is defined as follows:

$\varphi\left(f_{M R}\right)=\left\{\begin{array}{cc}-\frac{\chi}{\Re} e_{d} & \text { if } v>\frac{e_{d}}{\chi} \\ 0 & \text { if }-\frac{e_{d}}{\chi}<v<\frac{e_{d}}{\chi} \\ \frac{\chi}{\mathfrak{R}} e_{d} & \text { if } v<-\frac{e_{d}}{\chi}\end{array}\right.$

where, $e_{d}$ is the voltage applied to the MR damper. The value of $\varphi\left(f_{M R}\right)$ can be understood as $\Delta \varphi$. The value $\delta\left(\dot{f}_{M R}\right)$ is determined based on the upper bound $W$, which is defined as an offset of the damping force. This value can be understood as $\Delta \delta$. For the MR damper, the value $W$ is chosen considering the range of the minimum and maximum damping force shown in Figure 5(b). From Eqs. (60) and (61), the relationship between the damping force and main control input $u$ can be expressed as:

$$
F_{M R}=\tilde{\beta}_{\beta} u+\varphi\left(f_{M R}\right)+2 W=\tilde{\beta}_{\beta} u+\Delta \varphi+\Delta \delta
$$

From Eq. (63), the main control input $u$ is obtained as follows:

$$
u=\frac{F_{M R}-\Delta \varphi-\Delta \delta}{\tilde{\beta}_{\beta}}
$$

It is notice that in the practical applications of the proposed controller, from the resistance of the damper, the voltage $\vartheta$ will be changed to the current to generate the magnetic field which is 
required to achieve the desired damping force of the MR damper. It is remarked that the controllers proposed in $[23,36]$ will be used as a comparative model to verify the effectiveness of the proposed controller in this study.

\subsection{Simulation Results}

In this simulation, the performance of the proposed control declared in theorems 1 and 2 will be demonstrated. To verify the effectiveness of the proposed control, it is compared with other state -of-the-art controllers proposed in [23] (compared control 1) and [36] (compared control 2). Computer simulation for the above MR damper is carried out to evaluate the performance of the controllers. The principal parameters of the MR damper, seat suspension system and the converting values from voltage to current are given in [13]. The parameters of the dead-zone model areis firstly proposed, and then updated according to the learning progress of the fuzzy neural networks model. Two different excitations, i.e., the random bump road and random step wave road shown in Figure 6, are imposed to the seat suspension system. The maximum force of MR damper is designed to have $1000 \mathrm{~N}$ at $2 \mathrm{~A}$. The fuzzy neural networks model is adapting online with the centroid vector given in [13]. The selected parameters of the proposed controller, the controller proposed in [23] and the controller proposed in [36] are listed in Table 1, Table 2, and Table 3, respectively. In this simulation, the initial states for the dynamic states are assigned as $\left[\begin{array}{ll}0.035 & 2.5\end{array}\right]$ and $\left[\begin{array}{ll}0.035 & 2.5\end{array}\right]$ for random bump road, and random step wave bump, respectively. The initial states for the observer are assigned as $\left[\begin{array}{ll}0.035 & 0\end{array}\right]$ for two excitations.

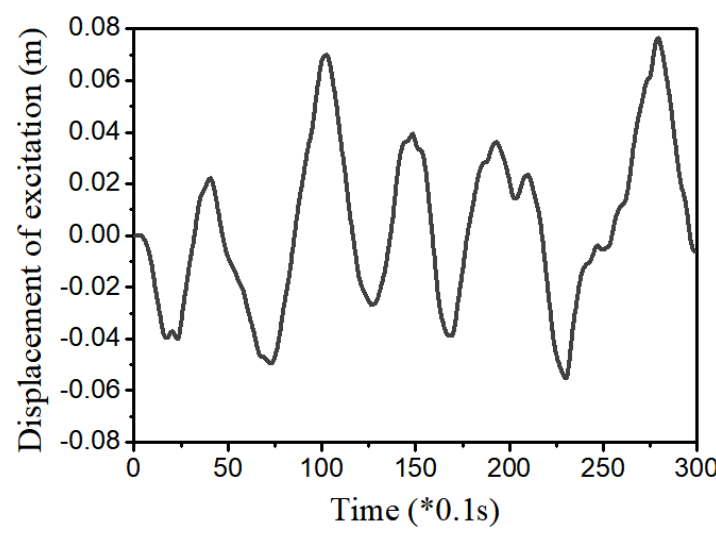

(a)

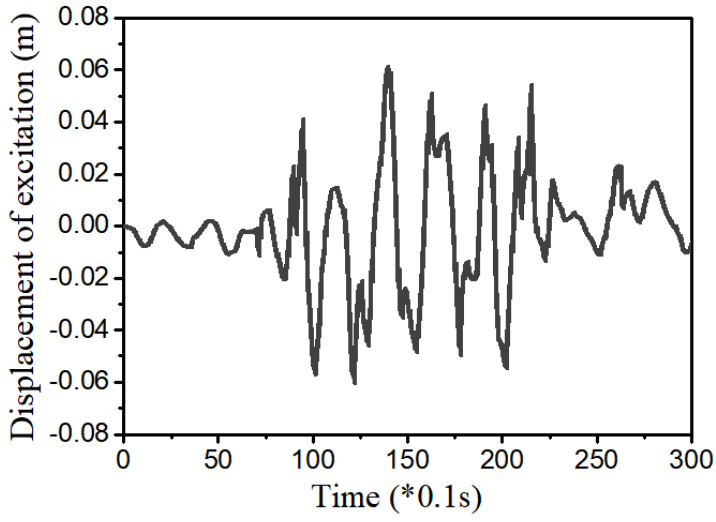

(b) 
Figure 6. Excitation signals: (a) random bump road, (b) random step wave road

Table 1. Parameters of simulation of proposed control

\begin{tabular}{|c|c|c|}
\hline Description & Symbol & Value \\
\hline Delayed time & $\tau$ & 0.1 \\
\hline Control function & $\delta(\dot{u})$ & 0.07 \\
\hline Dead-zone performance & $\theta^{+}, \theta^{-}$ & $0.08 ;-0.08$ \\
\hline Dead-zone performance & $\beta$ & 0.06 \\
\hline Dead-zone performance & $\beta_{\min }$ & 0.65 \\
\hline Dead-zone performance & $\beta_{\min 1}, \beta_{\min 2}$ & 0.65 \\
\hline Riccati-like constant & $\rho$ & 0.65 \\
\hline Riccati-like constant & $\rho_{1}, \rho_{2}$ & 0.65 \\
\hline Gain matrix & $\mathbf{K}_{i}=\left[\begin{array}{ll}K_{i 1} & K_{i 2}\end{array}\right]^{T}$ & $\mathbf{K}_{i}=\left[\begin{array}{ll}0.0001 & 1\end{array}\right]^{T}$ \\
\hline Constants & $\gamma_{f}, \gamma_{g}, \gamma_{D}, \gamma_{\beta}, \gamma_{v}$ & 700 \\
\hline Riccati-like matrix & $\mathbf{Q}$ & 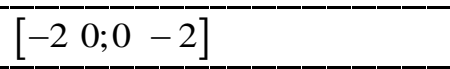 \\
\hline Riccati-like matrix & $\mathbf{R}$ & 0.1 \\
\hline
\end{tabular}

Table 2. Parameters of simulation of compared control 1 [23]

\begin{tabular}{l|l|l}
\hline \multicolumn{1}{c|}{ Description } & \multicolumn{1}{|c|}{ Symbol } & \multicolumn{1}{c}{ Value } \\
\hline Delayed time & $\tau$ & 0.1 \\
\hline Gain matrix & $\mathbf{K}_{i}=\left[\begin{array}{ll}K_{i 1} \quad K_{i 2}\end{array}\right]^{T}$ & $\mathbf{K}_{i}=\left[\begin{array}{ll}0.3 & 1\end{array}\right]^{T}$ \\
\hline Control function & $\delta(\dot{u})$ & 0.07 \\
\hline Dead-zone performance & $\beta$ & 0.06 \\
\hline Dead-zone performance & $\beta_{\text {min }}$ & 0.65 \\
\hline Dead-zone performance & $\theta^{+}, \theta^{-}$ & $0.08 ;-0.08$ \\
\hline Riccati-like constant & $\rho$ & 0.65 \\
\hline Riccati-like matrix & $\mathbf{Q}$ & $-20 ; 0-2]$ \\
\hline Riccati-like matrix & $\mathbf{R}$ & 0.1 \\
\hline
\end{tabular}

Table 3. Parameters of simulation of compared control 2 [36]

\begin{tabular}{c|l|l}
\hline \multicolumn{1}{c|}{ Description } & \multicolumn{1}{|c|}{ Symbol } & \multicolumn{1}{c}{ Value } \\
\hline Dead-zone performance & $\delta$ & 1 \\
\hline Dead-zone performance & $\omega$ & 1.7 \\
\hline Dead-zone performance & $a$ & 1000 \\
\hline
\end{tabular}

Simulation results will include the displacement of seat and force control input. These dynamic responses have important roles in evaluating the performance of the comparative controllers in terms of vibration suppression and energy consumption. The responses of the system under the inputs of the first proposed control (theorem 1) and the second proposed control (theorem 2) are shown in Figures 7 and 8 and Figures (9) and 10, respectively. The displacements of the seat 
under the controllers are shown in Figures 7 and 9. In Figure 7, the displacement of the seat under the inputs of the three controllers are fairly good. It is shown from Figure 7(a1) that the magnitude of vibration is significantly decreased after applying three controllers. The variations of control inputs and observers are shown in Figure 7(a2). This figure shows that the performances of the three controllers satisfy the observer's requirements. However, the performance of the proposed controller is better than that of the comparative controllers, as shown in Figure 7(a3) and Figure 7(a4). For the excitation of random pump road, the results are shown in Figures 7(b1), 7(b2), 7(b3), and 7(b4). It is clearly seen from Figure 7(b3) and 7(b4) that the proposed controller provides better performance than the comparative controllers. The control input $u$ is shown in Figure 8. It is clearly observed that the control energy of the proposed controller is less than that of the comparative controllers. This results directly prove that the proposed controller requires less power eensumption, butconsumption but provides better control performance. This merit is obtained based on the use of the adaptation law for the dead-zone problem, as discussed in remark 1. The flexible adaptation values of the update law $\tilde{\beta}_{\beta}$ in (25) and other adaptation laws of the proposed controller 1and the other laws-improve the system performance significantly. system with application of the proposed controller 1 . This is also show demonstrated that the affection effects of the dead-zone problem is limitedcan be suppressed.

For the excitation of random step wave road, the results are shown in Figures 9(a1)-9(a4). Similar results can be achieved for the random bump road, which verify better control performance of the proposed controller compared to other controllers. In addition, the vibration amplitude obtained by the proposed controller is less than that of the valued obtained by the comparative controllers, as shown in Figures $9(\mathrm{a} 3, \mathrm{a} 4, \mathrm{~b} 3, \mathrm{~b} 4)$. This results directly verify that the ride comfort of the driver can be enhanced by implementing the proposed controller. Figure 10 presents control inputs of the three controllers for two different road conditions. It can be seen that the input powers of the

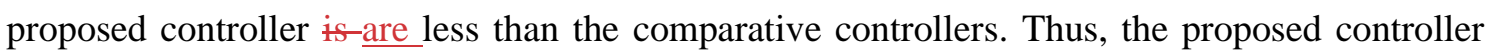
provides better vibration suppression performance and less power consumption than the comparative controllers. The updated values of the update law $\tilde{\beta}_{\beta}$ in (45), $\tilde{\mathbf{v}}$ in (46), and the other 
dead-zone and delayed time-problem with application of the proposed controller 2 . This is-also show that the affectiondemonstrated that the effects-of both dead-zone problem and delayed timeand time delay have been suppressed is limited.

From the above results, the compared control 1 is not flexible in calculation, as shown discussed in remarks $(1,2,8)$. It is also remained the main properties of the classical model of dead-zone. In the compared control 2, the simple calculation based on the classical dead-zone model is does still not solve the disadvantages as well. The compared control 2 is designed to enly seek the tminingtune the parameters following the time scale only. In addition, the sine function in the derived functions of tuning method is one of the obstructed ealeulationcalculations. Thist is considered based onfrom the property of the system which does is-not always follow a fixed model of dead-zone function. Then, the time convergence of the compared control 2 is also longer than the other controllers as shown in [36]. The simulation results verified are show that the proposed controller has overcome the disadvantages that the compared controls $(1,2)$ encountered; $\underline{\text { and }}$ decreased the effectsaffection of the dead-zone and time delay in minimallyto minimum.

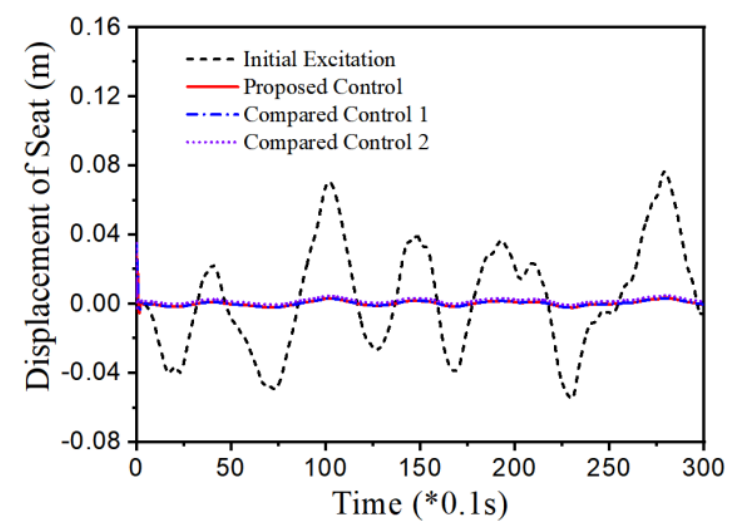

(a1)

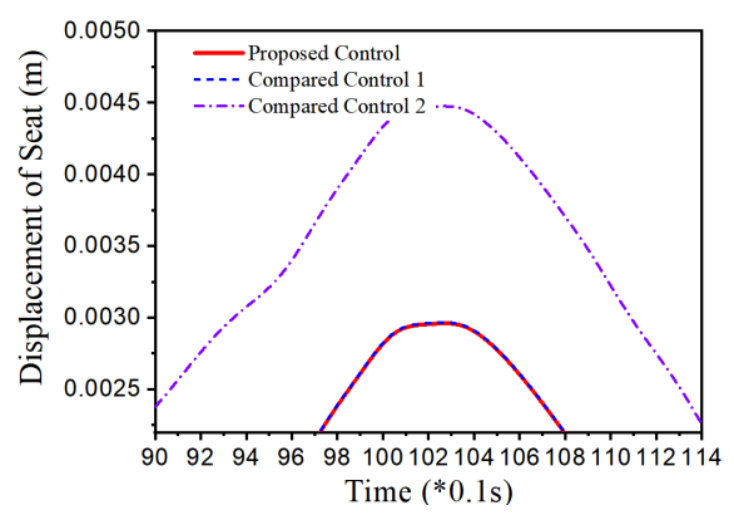

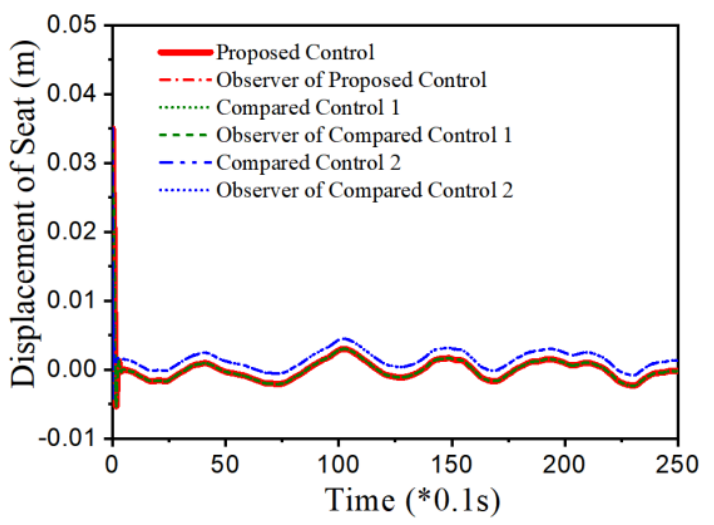

(a2)

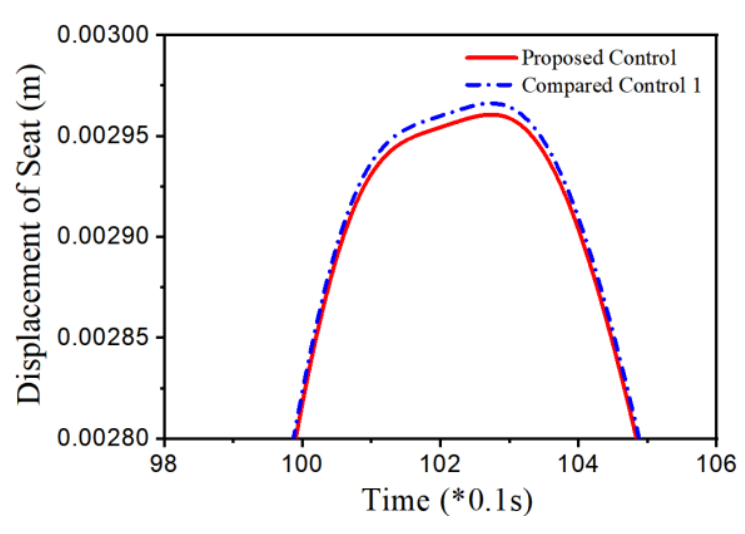


(a3)

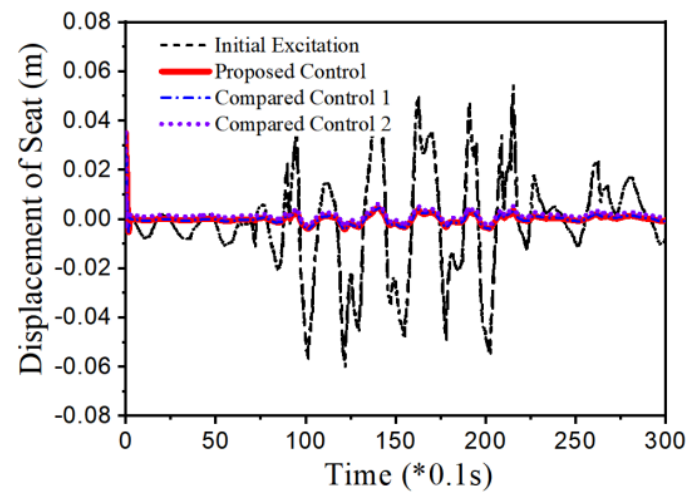

(b1)

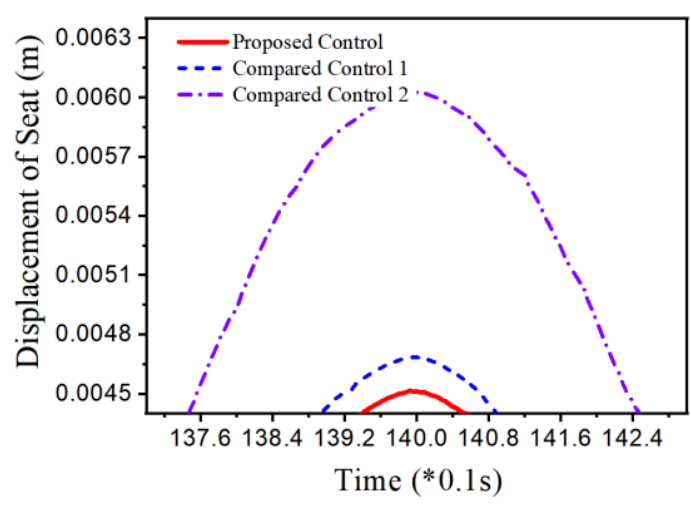

(b3) (a4)

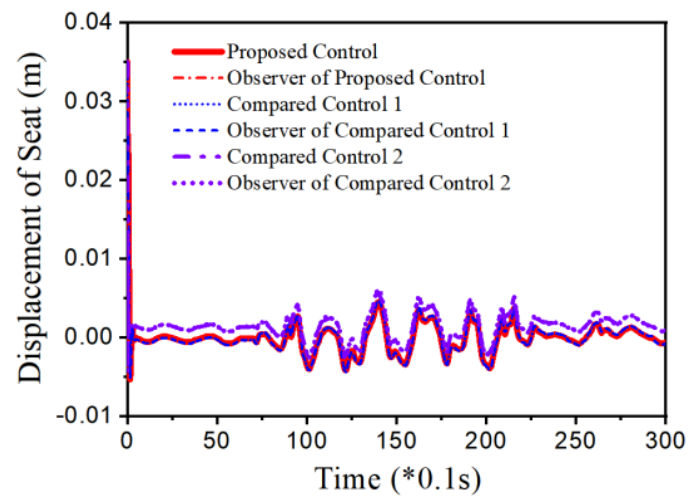

(b2)

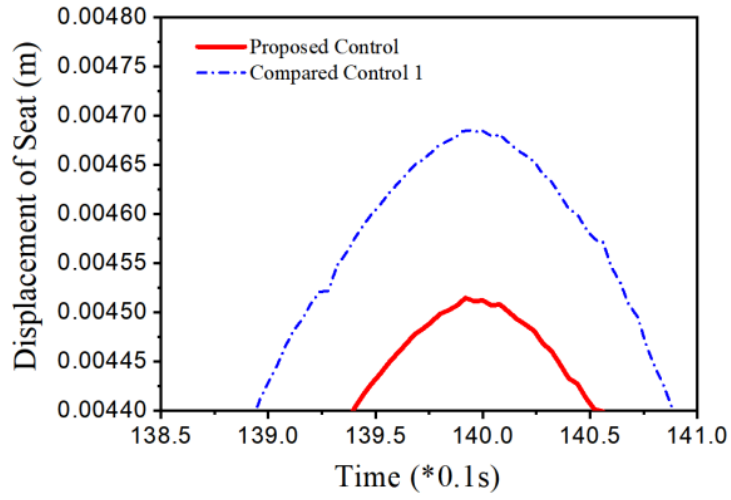

(b4)

Figure 7. Simulation results of the displacement at the seat $\left(x_{s}\right)$ : (a1) (a2)(a3)(a4) random bump road, (b1) (b2)(b3)(b4) random step wave road

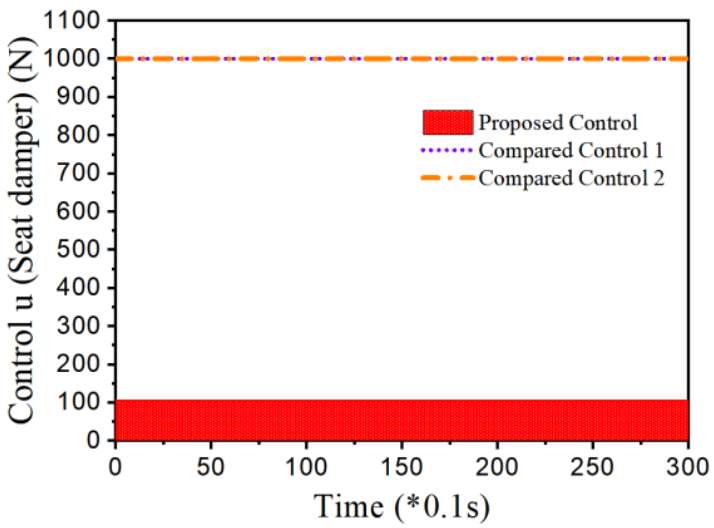

(a1)

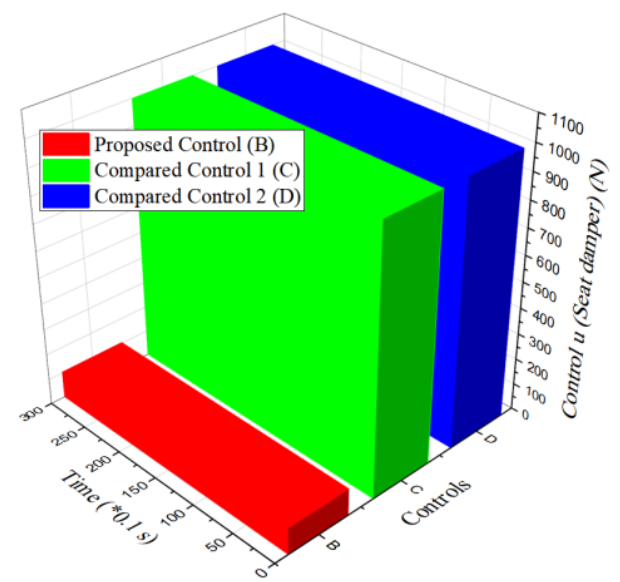

(a2) 


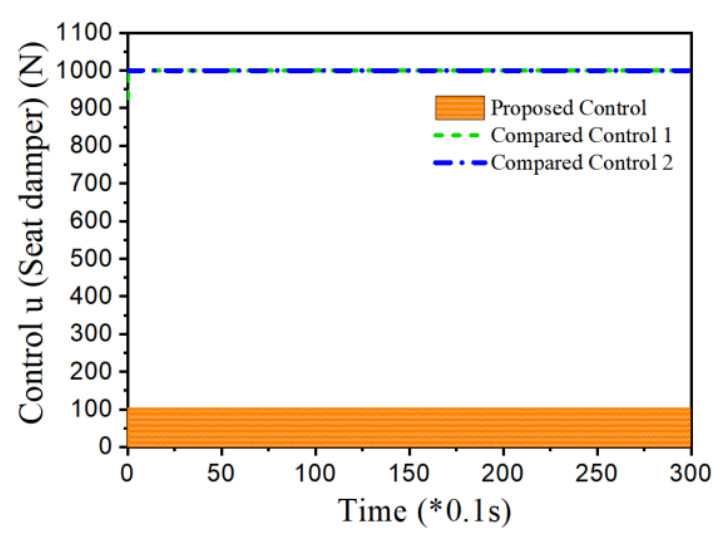

(b1)

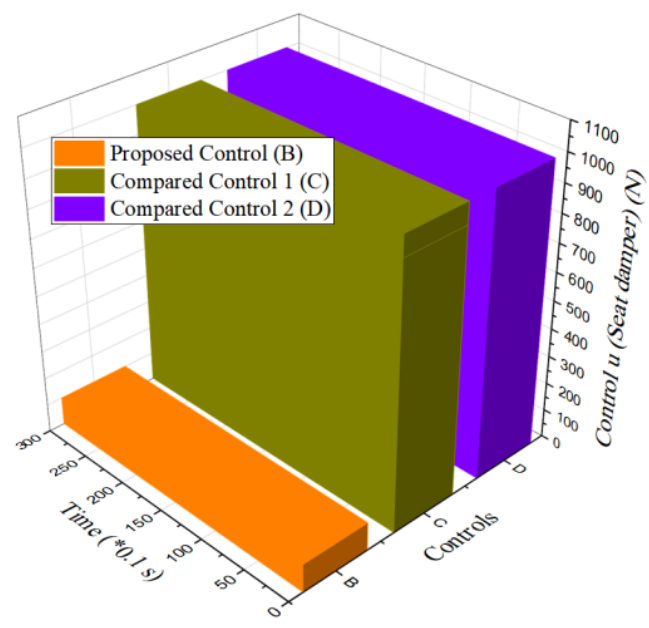

(b2)

Figure 8. Simulation results of force control $u$ : (a1)(a2) random bump road, (b1)(b2) random step wave road

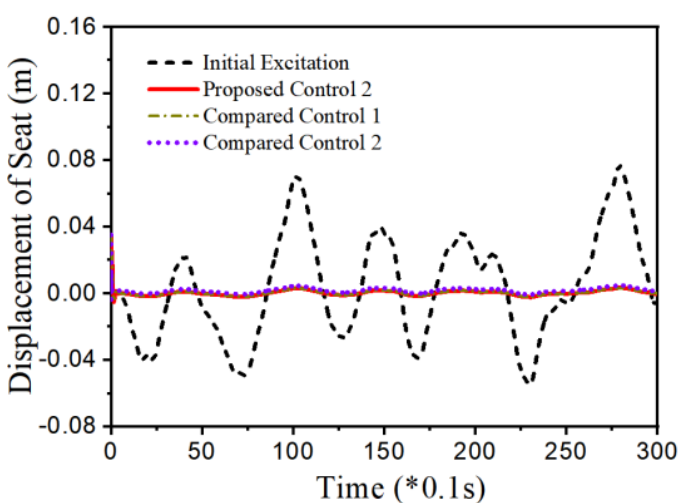

(a1)

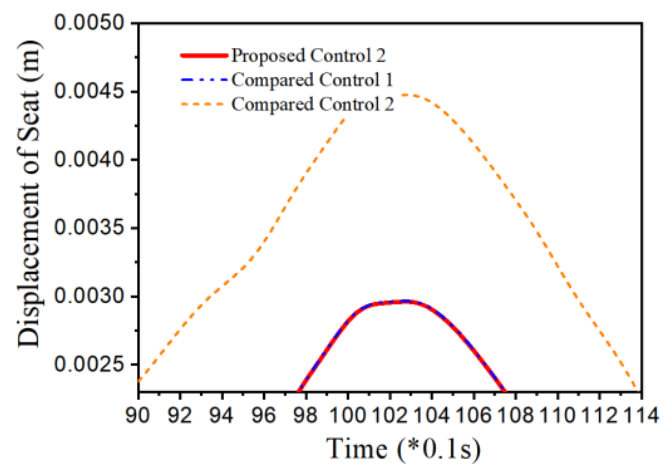

(a3)

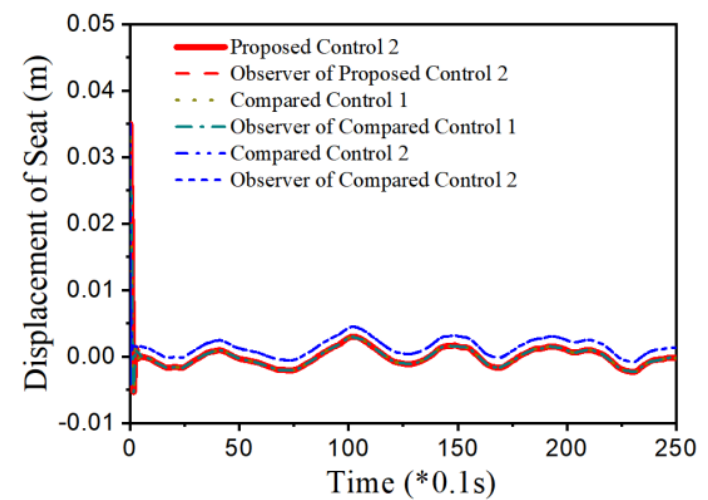

(a2)

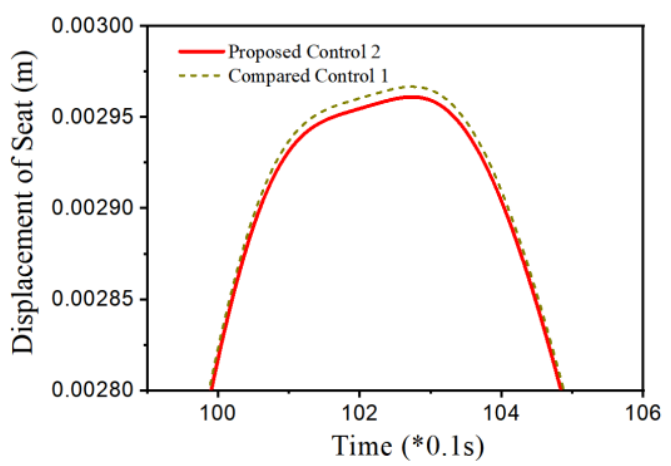

(a4) 


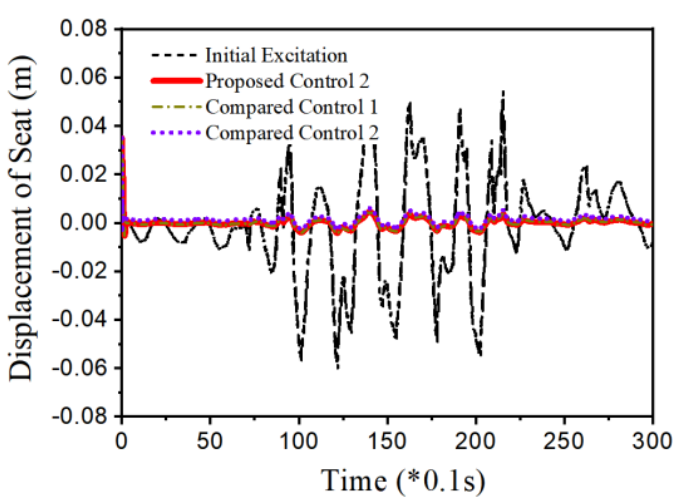

(b1)

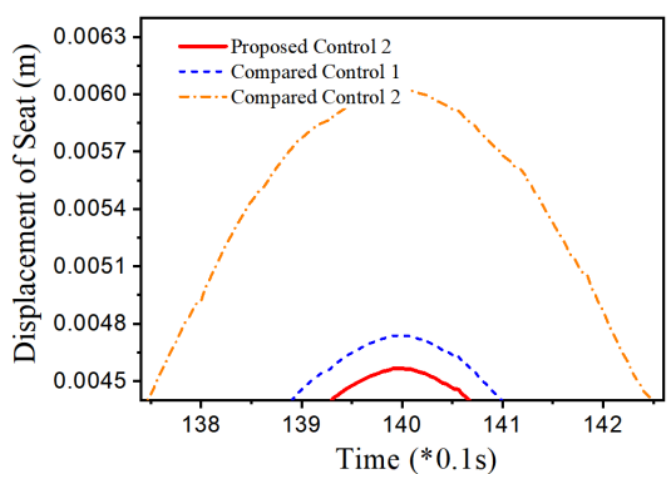

(b3)

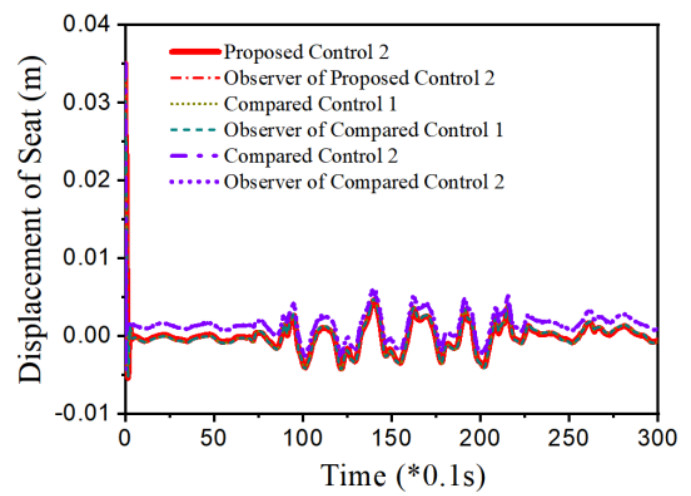

(b2)

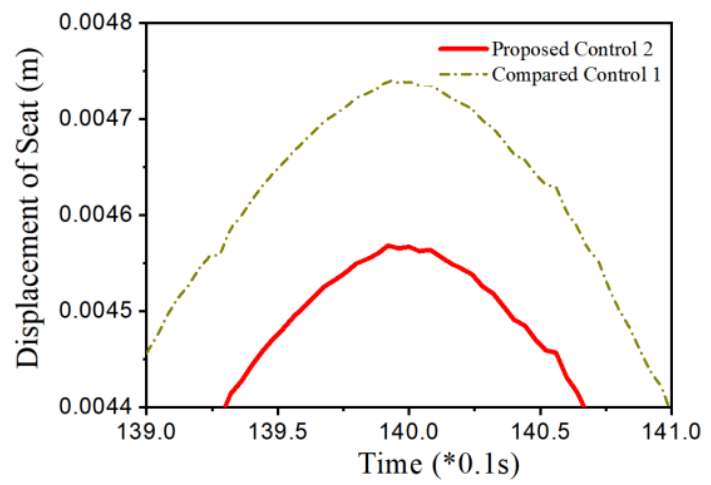

(b4)

Figure 9. Simulation results of the displacement at the seat $\left(x_{s}\right)$ : (a1) (a2) (a3)(a4)-random bump road; (b1) (b2) (b3) (b4)-random step wave road

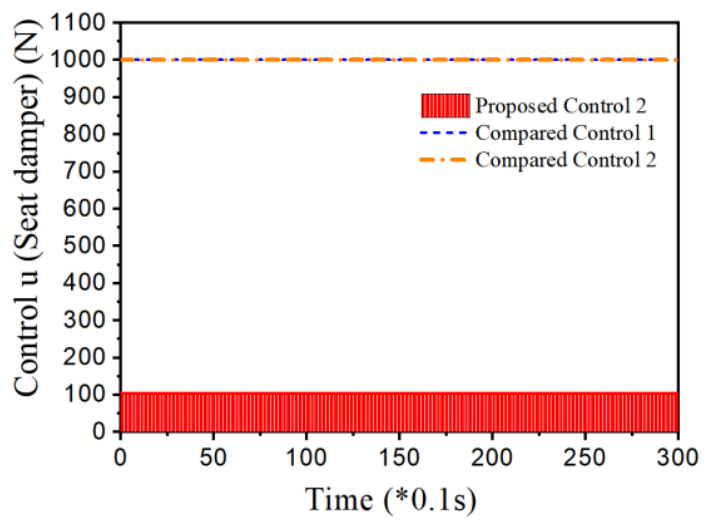

(a1)

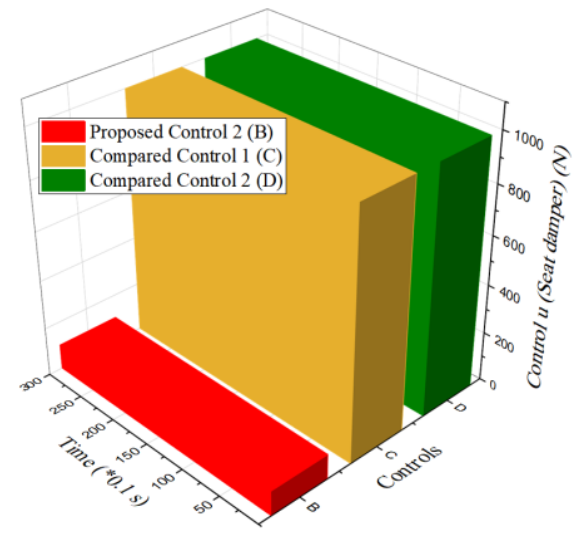

(a2) 


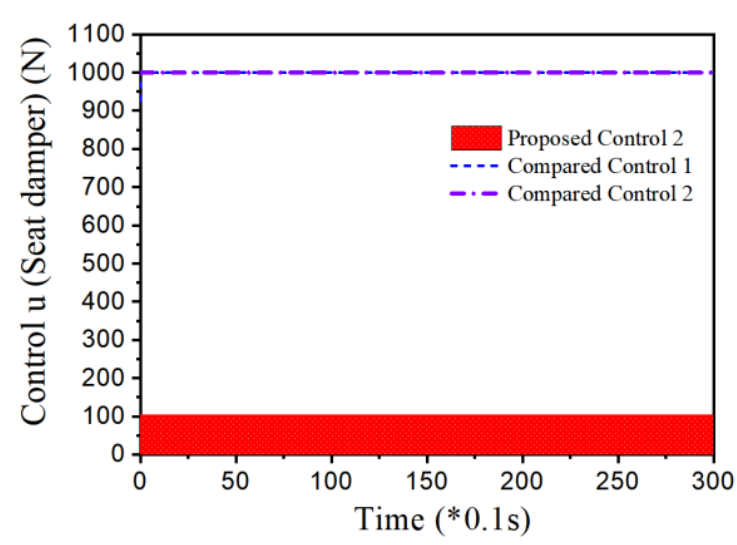

(b1)

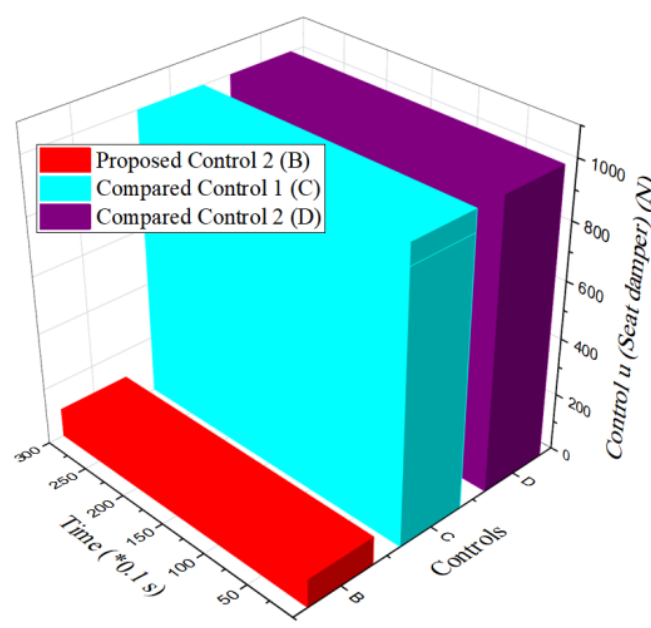

(b2)

Figure 10. Simulation results of force control $u$ : (a1)(a2) random bump road, (b1)(b2) random step wave road

\section{Conclusion}

In this study, a new adaptive controller was proposed based on a type 2 fuzzy model integrated with a neural network to resolve the dead-zone band and time delay issues of actuators. The proposed approach preserves many merits of many traditional controllers and adaptive techniques, including type 2 fuzzy model, adaptive control laws based on the sliding mode controller, $\mathrm{H}$ infinity control technique and modified Riccati-like equation. The new model of dead-zone band has overcome the disadvantage of the classical model of in control, especially applying for smart materials such as piezoelectric, magneto-rheological fluid. The proposed dead-zone model is als $\theta$ can be also applied for actuators made byusing materials possessing high time delay delayed time parameters. Two theorems have been presented: one for dead-zone problem, and the other for both dead-zone problem and time delayed time. The combination between the values of the deadzone band and the Riccati-like equation has improved the robustness properties of the controllers. The stability and convergence of the proposed controllers are proved rigorously based on Lyapunov stability criterion. The effectiveness of the proposed controllers areis validated through the computer simulation for a vehicle seat suspension system and comparing with two other stateof-the-art controllers. From the simulation results, it was demonstrated that the proposed controller provides better control performance with less power consumption compared to the two counterpart controllers. This performance enhancement indicates the better ride comfort of the vehicle seat. It should be mentioned that the proposed controller can be straightforwardly 
applicable for many control systems activated by smart material actuators such as shape memory alloys, piezoelectric materials, magneto_strictive materials and magnetorheological elastomers. The future works of this study will concentrate on extending the to applications -of the proposed control_approaches for real systems featuring smart materials such as seat suspension, energy harvesting system, mount vibration control.

\section{Acknowledgement}

This research is funded by Vietnam National Foundation for Science and Technology Development (NAFOSTED) under grant number 107.02-2020.13

\section{Declaration of conflicting interest}

The authors declare that there is no conflict of interest.

\section{References}

[1] Donya Ashtiani Haghighi, Saleh Mobayen, Design of an adaptive super-twisting decoupled terminal sliding mode control scheme for a class of fourth-order systems, ISA Transactions 75 (2018) 216-225

[2] Dong Liu, Guang-Hong Yang, Prescribed performance model-free adaptive integral sliding mode control for discrete-time nonlinear systems, IEEE Transactions on Neural Networks and Learning Systems (Early Access) (2018) Doi: 10.1109/TNNLS.2018.2881205

[3] Yan-Hui Jing and Guang-Hong Yang, Fuzzy adaptive fault-tolerant control for uncertain nonlinear systems with unknown dead-zone and unmodeled dynamics, IEEE Transactions on Fuzzy Systems (Early Access) (2019) Doi: 10.1109/TFUZZ.2019.2896844

[4] Abdelhamid Bounemeur, Mohamed chemachema, Najib Essounbouli, Indirect adaptive fuzzy fault-tolerant tracking control for MIMO nonlinear systems with actuator and sensor failures, ISA Transactions 79(2018) 45-61

[5] Xiaohong Cui, Huaguang Zhang, Yanhong Luo, Yanhong Luo, Adaptive dynamic programming for $\mathrm{H}$ infinity tracking design of uncertain nonlinear systems with disturbances and input constraints, Int J Adapt Control Signal Process 31(2017) 1567-1583 
[6] M. Davanipour, A.R. Khayatian, M. Dehghani, M.M. Arefi, A solution for enhancement of transient performance in nonlinear adaptive control: Optimal adaptive reset based on barrier Lyapunov function, ISA Transactions 80(2018) 169-175

[7] Mien Van, Xuan Phu Do, Optimal adaptive neural PI full-order sliding mode control for robust fault tolerant control of uncertain nonlinear system, European Journal of Control (in press) (2020) DOI: $10.1016 /$ j.ejcon.2019.12.005

[8] Do Xuan Phu, Van Mien, Seung Bok Choi, A novel adaptive gain of optimal sliding mode controller for linear time-varying systems, Applied Sciences 9(23) (2019) 5050

[9] Mien Van, Xuan Phu Do, Michalis Mavrovouniotis, Self-tuning fuzzy PID-nonsingular fast terminal sliding mode control for robust fault tolerant control of robot manipulators, ISA Transactions (in press) (2020) DOI: 10.1016/j.isatra.2019.06.017

[10] Yu Li, Chaoli Wang, Dengyu Liang, Xiaobin Li, Distributed adaptive leader-following consensus control of connected Lagrangian systems with input hysteresis quantization and timevarying control gain, Journal of the Franklin Institute 357 (2020) 942-977

[11] Hamed Rabiee, Mohammad Ataei, Mohsen Ekramian, Continuous nonsingular terminal sliding mode control based on adaptive sliding mode disturbance observer for uncertain nonlinear systems, Automatica 109 (2019) 108515

[12] Chenghu Jing, Hongguang Xu, Xinjian Niu, Adaptive sliding mode disturbance rejection control with prescribed performance for robotic manipulators, ISA Transactions 91 (2019) 41-51 [13] Do Xuan Phu, Jin-Hee An, Seung Bok Choi, A novel adaptive PID controller with application to vibration control of a semi-active vehicle seat suspension, Applied Sciences 7(10) (2017) 1055

[14] Meng Zhang, Peng Shi, Chao Shen, Zheng Guang Wu, Static output feedback control of switched nonlinear systems with actuator faults, IEEE Transactions on Fuzzy Systems (Early Access) (2019) Doi: 10.1109/TFUZZ.2019.2917177

[15] Meng Zhang, Chao Shen, Zheng Guang Wu, Dan Zhang, Dissipative filtering for switched fuzzy systems with missing measurements, IEEE Transactions on Cybernetics 50(5) (2020) 19311940 
[16] Yunkai Wu, Bin Jiang, Ningyun Lu, A descriptor system approach for estimation of incipient faults with application to high speed railway traction devices, IEEE Transactions on Systems, Man, and Cybernetics: Systems 49(10) (2019) 2108-2118

[17] Yunkai Wu, Bin Jiang, Yulong Wang, Incipient winding fault detection and diagnosis for squirrel-cage induction motors equipped on CRH trains, ISA Transactions 99 (2020) 488-495

[18] Weihai Zhang, Hang Su, Fuzzy adaptive control of nonlinear MIMO systems with unknown dead zone outputs, Journal of the Franklin Institute 355 (2018) 5690-5720

[19] Yu Zhang, Changchun Hua, Yafeng Li, Xinping Guan, Adaptive neural networks-based visual servoing control for manipulator with visibility constraint and dead-zone input, Neurocomputing 332 (2019) 44-55

[20] Wenxiang Deng,Jianyong Yao, Dawei Ma, Robust adaptive precision motion control of hydraulic actuators with valve dead-zone compensation, ISA Transactions 70 (2017) 269-278

[21] Kaixin Lu, Zhi Liu, Guanyu Lai, Yun Zhang, and C.L.Philip Chen, Adaptive fuzzy tracking control of uncertain nonlinear systems subject to actuator dead zone with piecewise time-varying parameters, IEEE Transactions on Fuzzy Systems (Early Access) (2018) Doi: 10.1109/TFUZZ.2018.2882170

[22] Yingyang Wang, Jianbo Hu, Improved prescribed performance control for air-breathing hypersonic vehicles with unknown dead-zone input nonlinearity, ISA Transactions 79 (2018) 95107

[23] Tzu-Sung Wu, Mansour Karkoub, Huiwei Wang, Ho-Sheng Chen, Ti-Hung Chen, Robust tracking control of MIMO underactuated nonlinear systems with dead-zone band and delayed uncertainty using an adaptive fuzzy control, IEEE Transactions on Fuzzy Systems 25(4) (2017) $905-918$

[24] Nilesh N.Karnik, Jerry M. Mendel, Centroid of a type 2 fuzzy set, Information Sciences 132 (2001) $195-220$

[25] H.M. Fayek, I. Elamvazuthi, N. Perumal, B. Venkatesh, A controller based on optimal type2 fuzzy logic: systematic design, optimization and real-time implementation, ISA Transactions 53 (2014) 1583-1591 
[26] Xianchang Wang, Xiaodong Liu, Lishi Zhang, A rapid fuzzy rule clustering method based on granular computing, Applied Soft Computing 24 (2014) 534-542

[27] Chia-Feng Juang and Yu-Wei Tsao, A self-evolving interval type-2 fuzzy neural network with online structure and parameter learning, IEEE Transactions on Fuzzy Systems 16(6) (2008) $1411-1424$

[28] Dongrui Wu, Approaches for reducing the computational cost of interval type-2 fuzzy logic systems: overview and comparisons, IEEE Transactions on Fuzzy Systems 21(1) (2013) 80-99

[29] Jerry M. Mendel, and Xinwang Liu, Simplified interval type-2 fuzzy logic systems, IEEE Transactions on Fuzzy Systems 21(6) (2013) 1056 - 1069

[30] Qilian Liang and Jerry M. Mendel, Interval type-2 fuzzy logic systems: theory and design, IEEE Transactions on Fuzzy Systems 8(5) (2000) 535-550

[31] N.M. Kwok, Q.P. Ha, T.H. Nguyen, J. Li, B. Samali, A novel hysteretic model for magnetorheological fluid dampers and parameter identification using particle swarm optimization, Sensors and Actuators A 132 (2006) 441-451

[32] Sang-Soo Kim, Yohji Okada, Variable resistance type energy regenerative damper using pulse width modulated step-up chopper, J. Vib. Acoust 124(1) (2002) 110-115

[33] Wuxi Shi, Observer-based indirect adaptive fuzzy control for SISO nonlinear systems with unknown gain sign, Neurocomputing 171 (2016) 1598-1605

[34] G. Ciccarella , M. Dalla Mora and A. Germani, A Luenberger-like observer for nonlinear systems, International Journal of Control 57(3) (1993) 537-556

[35] El Majdoub K, Ghani D, Giri F and Chaoui FZ, Adaptive semi-active suspension of quartervehicle with magnetorheological damper, Trans ASME, J Dynamic Systems, Measurement, Control 137 (2015) 021010-1-021010-12

[36] Guangru Shao, Andrew R. Teel, Ying Tan, Kun Zhi Liu, Rui Wang, Extremum seeking control with input dead-zone, IEEE Transactions on Automatic Control (Early acess) (2019) DOI: 10.1109/TAC.2019.2946427

[37] Peyman Yadmellat, Mehrdad R. Kermani, Adaptive control of a hysteretic magnetorheological robot actuator, IEEE/ASME Transactions on Mechatronics 21(3) (2016) $1336-1344$ 
[38] Bor Sen Chen, Ching Hsiang Lee, Yeong Chan Chang, Ho tracking design of uncertain nonlinear SISO systems: adaptive fuzzy approach, IEEE Transactions on Fuzzy Systems 4(1) (1996) 32-43

\section{Nomenclature}

\begin{tabular}{|c|c|}
\hline $\mathbf{H}_{f i}^{\mathrm{j}}(i=1, \ldots, n ; j=1, \ldots, m)$ & Fuzzy sets \\
\hline$m$ & Number of rules \\
\hline$a_{i}^{j}$ & Interval sets \\
\hline$g_{f}$ & Defuzzied output \\
\hline $\boldsymbol{\Phi}_{l}^{f}, \boldsymbol{\Phi}_{r}^{f}$ & Weighted firing strength vectors \\
\hline $\mathbf{f}(\mathbf{x}) \in R^{n}, \mathbf{g}(\mathbf{x}) \in R^{n}$ & Two unknown non-linear function vectors \\
\hline$\phi_{u}(t)=\beta u+\varphi(u)+\delta(\dot{u}), \phi_{u}(t) \in R^{1}$ & Control function \\
\hline$\beta$ & Parameter related slope of the dead-zone \\
\hline & performance (a chosen constant gain) \\
\hline$u$ & Control input \\
\hline$\varphi(u)$ & Dead-zone function \\
\hline$\Delta \varphi$ & Maximum boundary of dead-zone model \\
\hline$\delta(\dot{u})$ & Parameter related varied boundary of dead-zone \\
\hline & model \\
\hline$\Delta \delta$ & Maximum value of varied boundary \\
\hline $\mathbf{d}(t) \in R^{n}$ & An external disturbance vector \\
\hline$\delta \mathbf{d} \in R^{n}$ & Upper bound of $\mathbf{d}(\mathbf{t})$ \\
\hline $\mathbf{x}=\left[x_{1}, x_{2}, \ldots, x_{n}\right]^{T}=\left[x_{1}, \dot{x}_{1}, \ldots, x_{1}^{(n-1)}\right]^{T} \in R^{n}$ & The state vector of the system \\
\hline
\end{tabular}



$\Lambda, \Lambda_{m}(t)=\left[\begin{array}{ll}z_{m}(t) & 0\end{array}\right]^{T},\left|z_{m}(t)\right| \leq \delta z_{m}$
The nonlinear function related time-varying of the system
$\delta z_{m}$
Unknown positive constant
$\mathbf{x}_{d}=\left[x_{d 1}, x_{d 2}, \ldots, x_{d n}\right]^{T}=\left[x_{d 1}, \dot{x}_{d 1}, \ldots, x_{d 1}^{(n-1)}\right]^{T} \in R^{n}$ Desired value
$\mathbf{e}=\mathbf{x}-\mathbf{x}_{d}$
Error vector
$\mathbf{K}=\operatorname{diag}\left\{\begin{array}{lll}\mathbf{K}_{1} & \cdots & \mathbf{K}_{n}\end{array}\right\}$
Feedback gain matrix
$\mathbf{K}_{i}=\left[\begin{array}{ll}K_{i 1} & K_{i 2}\end{array}\right]^{T}$
Chosen vector such that all roots of the characteristic
polynomial
of

$\mathbf{A}_{i}=\mathbf{A}-\mathbf{B K}_{i}^{T}, i=1, \ldots, n$ are in the open left half plane

$\hat{\mathbf{f}}(\mathbf{x})=\boldsymbol{\Xi}_{f}(x) \boldsymbol{\Phi}_{f}, \hat{\mathbf{g}}(\mathbf{x})=\boldsymbol{\Xi}_{g}(x) \boldsymbol{\Phi}_{g}$

Fuzzified functions of $\mathbf{f}(\mathbf{x})$ and $\mathbf{g}(\mathbf{x})$, respectively

$\Delta \mathbf{f}(\mathbf{x}), \Delta \mathbf{g}(\mathbf{x})$

The minimum approximation errors

$\mathbf{D}=\mathbf{\Delta} \mathbf{f}+\Delta \mathbf{g}(\beta u+\varphi(u)+\delta(\dot{u}))$

The minimum approximation error related deadzone phenomenon

$u_{1}$

The equivalent control

$u_{2}$

The first robustness control related the Riccati-

like equation

$u_{3}$

The second robustness control related the deadzone band, the Riccati-like equation and the minimum approximation error $\mathbf{D}$ of the system

$u_{4}$ The third robustness control related the delayed model

Parameter related delayed time

$\mathbf{R}$

The robust $\mathrm{H}$ infinity technique gain 


\begin{tabular}{|c|c|}
\hline$\beta_{\min 1}$ & Parameter related dead-zone model \\
\hline$\beta_{\min 2}$ & Parameter related delayed model \\
\hline$\gamma_{f}, \gamma_{g}, \gamma_{D}, \gamma_{\beta}, \gamma_{v}$ & Chosen constants \\
\hline$m_{s}$ & Mass of seat \\
\hline$m_{1}$ & Mass of driver \\
\hline$k_{s}$ & Stiffness coefficient of seat \\
\hline$k_{1}$ & Stiffness coefficient of torso \\
\hline$c_{s}$ & Damping coefficient of seat \\
\hline$c_{1}$ & Damping coefficient of torso \\
\hline$x_{11}, x_{22}, x_{33}, x_{44}$ & Variables related $x_{s}$ and $x_{1}$ of the system \\
\hline$f_{M R}$ & Theoretical damping force of the damper \\
\hline$\chi$ & $\begin{array}{l}\text { The actuation constant related viscous } \\
\text { coefficient }(\mathrm{V} . \mathrm{s} / \mathrm{m})\end{array}$ \\
\hline$\Re$ & The resistance of the circuit of damper $(\Omega)$ \\
\hline$v$ & The velocity of damper $(\mathrm{m} / \mathrm{s})$ \\
\hline$e_{d}$ & The voltage applied to the damper \\
\hline$\Gamma$ & $\begin{array}{l}\text { The upper boundary } \Gamma \text { which is defined as an } \\
\text { offset of the damping force, and this value can } \\
\text { be seen as } \Delta \delta\end{array}$ \\
\hline$\vartheta$ & The voltage to be applied to MR damper \\
\hline
\end{tabular}

